\title{
Free Vibration Analysis of Centrifugally Stiffened Non Uniform Timoshenko Beams
}

\author{
Diana V. Bambill, Daniel H. Felix, Raúl E. Rossi and Alejandro R. Ratazzi \\ Universidad Nacional del Sur, UNS, Departamento de Ingeniería, \\ Instituto de Mecánica Aplicada, IMA, \\ Consejo Nacional de Investigaciones Científicas y Técnicas, CONICET, \\ Argentina
}

\section{Introduction}

Rotating beams - like structures are widely used in many engineering fields and are of great interest as they can be used to model blades of wind turbines, helicopter rotors, robotic manipulators, turbo-machinery and aircraft propellers. The governing differential equations of motion in free vibration of a non-uniform rotating Timoshenko beam, with general elastic restraints at the ends are solved using the differential quadrature method, (Bellman \& Roth, 1986; Felix et al., 2008, 2009). The equations of motion are derived to include the effects of shear deformation, rotary inertia, hub radius, ends elastically restrained and non-uniform variation of the cross-sectional area of the beam. The presence of a centrifugal force due to the rotational motion is considered as Banerjee has developed, using Hamilton's principle to capture the centrifugal stiffening arising in fast rotating structures, (Banerjee, 2001). With the proposed model, a great number of different situations are admitted to be solved. Particular cases with classical restraints can be deduced for limiting values of the rigidities. Also step changes in cross-section are considered (Naguleswaran, 2004).

The natural vibration frequencies and mode shapes of rotating beams have been a topic of interest and have received considerable attention. A large number of researchers have studied the dynamic behavior of rotating uniform or tapered Euler-Bernoulli beams. (Yang el al., 2004; Özdemir \& Kaya, 2006; Lin \& Hsiao, 2001). Banerjee derived the dynamic stiffness matrix of a rotating Bernoulli-Euler beam using the Frobenius method of solution in power series and he includes the presence of an axial force at the outboard end of the beam in addition to the existence of the usual centrifugal (Banerjee, 2000).

Not so many studies have tackled the problem of rotating beams taking into account rotary inertia, shear deformation and their combined effects, hub radius and ends elastically restrained, (Bambill et al., 2010). In applications where the rotary inertia and the shear deformation effects are not significant, an analysis based on the Euler-Bernoulli beam theory can be used. However, Timoshenko theory allows describing the vibration of short beams, sandwich composite beams or high modes of a slender beam, (Rossi et al., 1991; Seon et al., 1999). (Banerjee et al., 2006) investigated the free bending vibration of rotating tapered Timoshenko beams by the dynamic stiffness method. (Ozgumus \& Kaya, 2010) used the Differential Transform Method for free vibration analysis of a rotating, tapered Timoshenko beam. 
The finite element method was used by (Hodges \& Rutkowski, 1981). (Vinod et al., 2007) presented a study about spectral finite element formulation for a rotating beam subjected to small duration impact. (Gunda \& Ganguli, 2008) developed a new beam finite element whose basis functions were obtained by the exact solution of the governing static homogenous differential equation of a stiff string, which resulted from an approximation in the rotating beam equation. (Singh et al., 2007) used the Genetic Programming to create an approximate model of rotating beams. (Gunda et al., 2007) introduced a low degree of freedom model for dynamic analysis of rotating tapered beams based on a numerically efficient superelement, developed using a combination of polynomials and Fourier series as shape functions. (Kumar \& Ganguli, 2009) looked for rotating beams whose eigenpair, frequency and mode-shape, is the same as that of uniform non rotating beams for a particular mode. An interesting paper (Ganesh \& Ganguli, 2011) presented physics based basis function for vibration analysis of high speed rotating beams using the finite element method. The basis function gave rise to shape functions which depend on position of the element in the beam, material, geometric properties and rotational speed of the beam.

The present study tries to provide not only solutions for practical engineering situations but they also may be useful as benchmark for comparing other numerical models. The proposed differential quadrature method, offers a useful and accurate procedure for the solution of linear and non linear partial differential equations. It was used by Bellman in the 1970's. He used this method to calculate the natural frequencies of transverse vibration of a rotating cantilever beam. (Bellman \& Casti, 1971). Other authors have used the differential quadrature method and recognized it as an effective technique for solving this kind of problems, (Bert \& Malik, 1996; Shu \& Chen, 1999; Choi et al., 2000; Liu \& Wu, 2001; Shu, 2000).

Numerical results are obtained for the natural frequencies of transverse vibration and the mode shapes of rotating beams considering the elastic restraints, with non uniform variation of the cross-sectional area. Some of those cases have also been solved using the finite element method, and the sets of results are in excellent agreement.

\section{Theory}

Figure 1 shows the rotating tapered beam considered in the present paper. The beam could have step jumps in cross section and rotates at speed $\bar{\eta}$. The $\bar{X}$-axis coincides with the centroidal axis of the beam, the $\bar{Y}$-axis is parallel with the axis of rotation and the $\bar{Z}$-axis lies in the plane of rotation. $L$ is the length of the beam, $L_{k}$ is the length of the segment $k$ and $L_{d}$ is the length of the last segment of the beam. The displacement in the $\bar{Y}$ direction is denoted as $\bar{w}$ and the section rotation is denoted as $\bar{\psi}$. Only displacements in the $\bar{X}-\bar{Y}$ plane are taken into account and the Coriolis effects are not considered.

The centrifugal force of a beam element at a distance $\bar{R}_{k}+\bar{x}_{k}$ from the axis of rotation can be expressed as

$$
d \bar{F}_{k}=\bar{\eta}^{2}\left(\bar{R}_{k}+\bar{x}_{k}\right) d m
$$

where $d m=\rho A_{k}\left(\bar{x}_{k}\right) d \bar{x}_{k}$ is its mass, with $\rho$ the mass density of material, and $A_{k}\left(\bar{x}_{k}\right)$, is the cross-sectional area at $\bar{x}_{k}$. Figure 2 . The centrifugal force $\bar{N}_{k}\left(\bar{x}_{k}\right)$ generated by $\bar{\eta}$ is

$$
d \bar{N}_{k}\left(\bar{x}_{k}\right)=\bar{\eta}^{2} \rho\left(\bar{R}_{k}+\bar{x}_{k}\right) A_{k}\left(\bar{x}_{k}\right) d \bar{x}_{k}
$$


The total axial force at the cross section located at $\bar{R}_{k}+\bar{x}_{k}$ is

$$
\bar{N}_{k}\left(\bar{x}_{k}\right)=\bar{\eta}^{2} \rho \int_{\bar{x}_{k}}^{L_{k}}\left(\bar{R}_{k}+\bar{x}_{k}\right) A_{k}\left(\bar{x}_{k}\right) d \bar{x}_{k}+\bar{F}_{k+1}=\bar{\eta}^{2} \rho\left(\bar{R}_{k} \int_{\bar{x}_{k}}^{L_{k}} A_{k}\left(\bar{x}_{k}\right) d \bar{x}_{k}+\int_{\bar{x}_{k}}^{L_{k}} A_{k}\left(\bar{x}_{k}\right) \bar{x}_{k} d \bar{x}_{k}\right)+\bar{F}_{k+1}
$$

$\bar{F}_{k+1}$ is the outboard force at the end of the segment $k$, due to the adjacent segments $k+1$ to $d$.

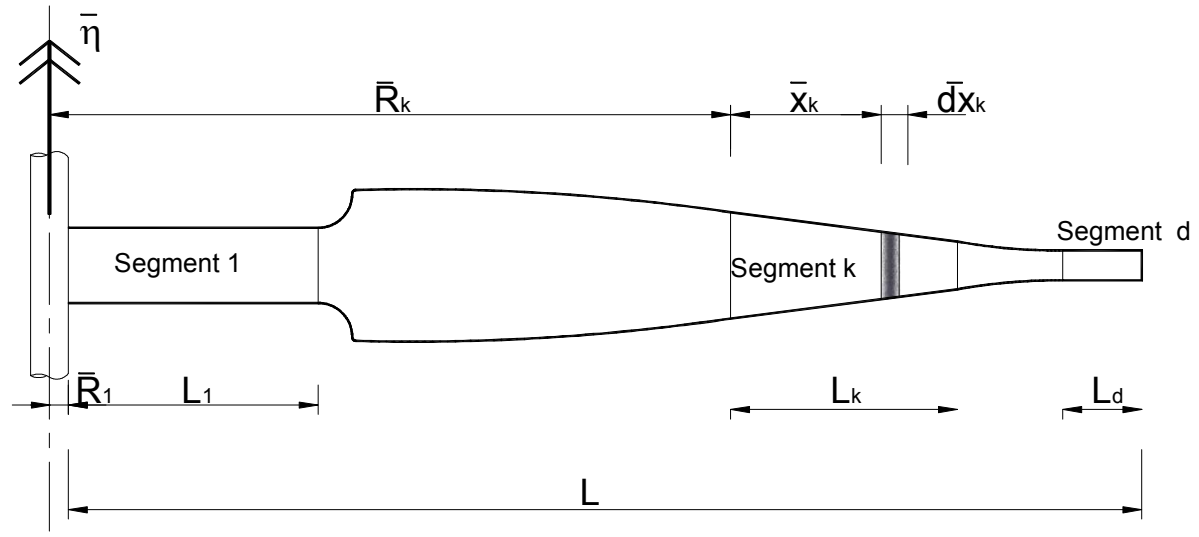

Fig. 1. Rotating beam model

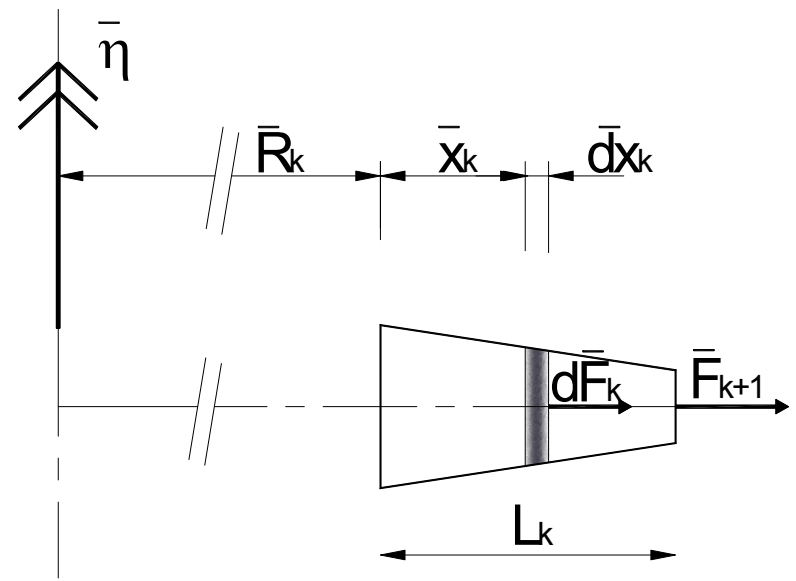

Fig. 2. Rotating beam segment $k$ of length $L_{k}$

Finally, the tensile force can be written as

$$
\bar{N}_{k}\left(\bar{x}_{k}\right)=\bar{\eta}^{2} \rho\left(\bar{R}_{k} V_{k}\left(L_{k}\right)+\Phi_{k}\left(L_{k}\right)-\bar{R}_{k} V_{k}\left(\bar{x}_{k}\right)-\Phi_{k}\left(\bar{x}_{k}\right)\right)+\bar{F}_{k+1}
$$

with 


$$
V_{k}\left(\bar{x}_{k}\right)=\int_{0}^{\bar{x}_{k}} A_{k}\left(\bar{x}_{k}\right) d \bar{x}_{k} ; \Phi_{k}\left(\bar{x}_{k}\right)=\int_{0}^{\bar{x}_{k}} A_{k}\left(\bar{x}_{k}\right) \bar{x}_{k} d \bar{x}_{k}
$$

The expressions for shear force and bending moment at an instant $t$ in the rotating beam are

$$
\begin{gathered}
\bar{Q}_{k}^{*}\left(\bar{x}_{k}, t\right)=\bar{N}_{k}\left(\bar{x}_{k}\right) \frac{\partial \bar{w}_{k}\left(\bar{x}_{k}, t\right)}{\partial \bar{x}_{k}}+\kappa G A_{k}\left(\bar{x}_{k}\right)\left(\frac{\partial \bar{w}_{k}\left(\bar{x}_{k}, t\right)}{\partial \bar{x}_{k}}-\bar{\psi}_{k}\left(\bar{x}_{k}, t\right)\right) \\
\bar{M}_{k}^{*}\left(\bar{x}_{k}, t\right)=E I_{k}\left(\bar{x}_{k}\right) \frac{\partial \bar{\psi}_{k}\left(\bar{x}_{k}, t\right)}{\partial \bar{x}_{k}}
\end{gathered}
$$

where $I_{k}\left(\bar{x}_{k}\right)$ is the second moment of area of the beam cross-section; $t$ the time; $\bar{w}_{k}(\bar{x}, t)$ the transverse displacement; $\bar{\psi}_{k}(\bar{x}, t)$ the section rotation; $E$ the Young's modulus; $v$ the Poisson's ratio; $G=E / 2(1+v)$ the shear modulus and $\kappa$ is the shear factor.

The governing differential equations of motion of a rotating Timoshenko beams (Banerjee, 2001) are:

$$
\begin{gathered}
\frac{\partial \bar{Q}_{k}^{*}\left(\bar{x}_{k}, t\right)}{\partial \bar{x}_{k}}=\rho A_{k}\left(\bar{x}_{k}\right) \frac{\partial^{2} \bar{w}_{k}\left(\bar{x}_{k}, t\right)}{\partial t^{2}} \\
\bar{Q}_{k}^{*}\left(\bar{x}_{k}, t\right)-N_{k}\left(\bar{x}_{k}\right) \frac{\partial \bar{w}_{k}\left(\bar{x}_{k}, t\right)}{\partial \bar{x}_{k}}+\frac{\partial \bar{M}_{k}^{*}\left(\bar{x}_{k}, t\right)}{\partial \bar{x}_{k}}+\rho I_{k}\left(\bar{x}_{k}\right) \bar{\eta}^{2} \bar{\psi}_{k}\left(\bar{x}_{k}, t\right)=\rho I_{k}\left(\bar{x}_{k}\right) \frac{\partial^{2} \bar{\psi}_{k}\left(\bar{x}_{k}, t\right)}{\partial t^{2}}
\end{gathered}
$$

Assuming simple harmonic oscillation

$$
\bar{w}_{k}\left(\bar{x}_{k}, t\right)=\bar{W}_{k}\left(\bar{x}_{k}\right) e^{i \omega t} ; \bar{\psi}_{k}\left(\bar{x}_{k}, t\right)=\bar{\Psi}_{k}\left(\bar{x}_{k}\right) e^{i \omega t}
$$

where $\omega$ is the circular frequency in radian per second.

The bending moment and the shear force are expressed as

$$
\bar{Q}_{k}^{*}\left(\bar{x}_{k}, t\right)=\bar{Q}_{k}\left(\bar{x}_{k}\right) e^{i \omega t} ; \bar{M}_{k}^{*}\left(\bar{x}_{k}, t\right)=\bar{M}_{k}\left(\bar{x}_{k}\right) e^{i \omega t}
$$

where

$$
\bar{Q}_{k}\left(\bar{x}_{k}\right)=\left(\bar{N}_{k}\left(\bar{x}_{k}\right)+\kappa G A_{k}\left(\bar{x}_{k}\right)\right) \frac{d \bar{W}_{k}\left(\bar{x}_{k}\right)}{d \bar{x}_{k}}-\kappa G A_{k}\left(\bar{x}_{k}\right) \bar{\Psi}_{k}\left(\bar{x}_{k}\right) ; \bar{M}_{k}\left(\bar{x}_{k}\right)=E I_{k}\left(\bar{x}_{k}\right) \frac{d \bar{\Psi}_{k}\left(\bar{x}_{k}\right)}{d \bar{x}_{k}}
$$

Substituting equations (9-10) into equations (8), the equations of motion for the free vibration of the segment $k$ of the rotating beam result in:

$$
\begin{gathered}
-\frac{d \bar{Q}_{k}\left(\bar{x}_{k}\right)}{d \bar{x}_{k}}=\rho A_{k}\left(\bar{x}_{k}\right) \omega^{2} \bar{W}_{k}\left(\bar{x}_{k}\right) \\
-\bar{Q}_{k}\left(\bar{x}_{k}\right)+\bar{N}_{k}\left(\bar{x}_{k}\right) \frac{d \bar{W}_{k}\left(\bar{x}_{k}\right)}{d \bar{x}_{k}}-\frac{d \bar{M}_{k}\left(\bar{x}_{k}\right)}{d \bar{x}_{k}}-\rho I_{k}\left(\bar{x}_{k}\right) \bar{\eta}^{2} \bar{\Psi}_{k}\left(\bar{x}_{k}\right)=\rho I_{k}\left(\bar{x}_{k}\right) \omega^{2} \bar{\Psi}_{k}\left(\bar{x}_{k}\right)
\end{gathered}
$$


Replacing equations (11) into equations (12), the differential equations of motion become:

$$
\begin{gathered}
-\frac{d \bar{N}_{k}\left(\bar{x}_{k}\right)}{d \bar{x}_{k}} \frac{d \bar{W}_{k}\left(\bar{x}_{k}\right)}{d \bar{x}_{k}}-\bar{N}_{k}\left(\bar{x}_{k}\right) \frac{d^{2} \bar{W}_{k}\left(\bar{x}_{k}\right)}{d \bar{x}_{k}^{2}}-\kappa G A_{k}\left(\bar{x}_{k}\right)\left(\frac{d^{2} \bar{W}_{k}\left(\bar{x}_{k}\right)}{d \bar{x}_{k}^{2}}-\frac{d \bar{\Psi}_{k}\left(\bar{x}_{k}\right)}{d \bar{x}}\right)- \\
\kappa G \frac{d A_{k}\left(\bar{x}_{k}\right)}{d \bar{x}_{k}}\left(\frac{d \bar{W}_{k}\left(\bar{x}_{k}\right)}{d \bar{x}_{k}}-\bar{\Psi}_{k}\left(\bar{x}_{k}\right)\right)=\rho A_{k}\left(\bar{x}_{k}\right) \omega^{2} \bar{W}_{k}\left(\bar{x}_{k}\right) \\
-\kappa G A_{k}\left(\bar{x}_{k}\right)\left(\frac{d \bar{W}_{k}\left(\bar{x}_{k}\right)}{d \bar{x}_{k}}-\bar{\Psi}_{k}\left(\bar{x}_{k}\right)\right)-E I_{k}\left(\bar{x}_{k}\right) \frac{d^{2} \bar{\Psi}_{k}\left(\bar{x}_{k}\right)}{d \bar{x}_{k}^{2}}- \\
E \frac{d I_{k}\left(\bar{x}_{k}\right)}{d \bar{x}_{k}} \frac{d \bar{\Psi}_{k}\left(\bar{x}_{k}\right)}{d \bar{x}_{k}}-\rho I_{k}\left(\bar{x}_{k}\right) \bar{\eta}^{2} \bar{\Psi}_{k}\left(\bar{x}_{k}\right)=\rho I_{k}\left(\bar{x}_{k}\right) \omega^{2} \bar{\Psi}_{k}\left(\bar{x}_{k}\right)
\end{gathered}
$$

The term $\rho I_{k}\left(\bar{x}_{k}\right) \bar{\eta}^{2} \bar{\Psi}_{k}\left(\bar{x}_{k}\right)$ included in equation (13.b) was introduced by Banerjee, 2001. This term generates more realistic results especially for high rotational speeds, $\bar{\eta}^{2}$.

The conditions for displacements and forces between adjacent segments, $k$ and $k+1$, are:

$$
\begin{aligned}
& \bar{W}_{k}\left(L_{k}\right)-\bar{W}_{k+1}(0)=0 ; \quad \bar{\Psi}_{k}\left(L_{k}\right)-\bar{\Psi}_{k+1}(0)=0 \\
& \bar{Q}_{k}\left(L_{k}\right)-\bar{Q}_{k+1}(0)=0 ; \quad \bar{M}_{k}\left(L_{k}\right)-\bar{M}_{k+1}(0)=0
\end{aligned}
$$

Figure 3 shows the beam elastically restrained at both ends.

The boundary conditions of the beam at its ends are, for the first segment $k=1$, at $\overline{x_{1}}=0$ :

$$
\bar{Q}_{1}(0)-\bar{K}_{W 1} \bar{W}_{1}(0)=0 ; \bar{M}_{1}(0)-\bar{K}_{\Psi 1} \bar{\Psi}_{1}(0)=0
$$

and for the last segment $k=d$, at $\overline{x_{d}}=L_{d}$ :

$$
\bar{Q}_{d}\left(L_{d}\right)-\bar{K}_{W d} \bar{W}_{d}(0)=0 ; \bar{M}_{d}\left(L_{d}\right)-\bar{K}_{\Psi d} \bar{\Psi}_{d}(0)=0
$$

The four spring constants are denoted as: $\bar{K}_{W 1}, \bar{K}_{W d}, \bar{K}_{\Psi 1}, \bar{K}_{\Psi d}$.

The expressions and parameters in dimensionless form are defined as follows:

$$
\begin{gathered}
\Omega^{2}=\frac{\rho A_{1}(0)}{E I_{1}(0)} L^{4} \omega^{2} ; \eta^{2}=\frac{\rho A_{1}(0)}{E I_{1}(0)} L^{4} \bar{\eta}^{2} ; \\
x=\frac{\bar{x}_{k}}{L_{k}} ; l_{k}=\frac{L_{k}}{L} ; r_{k}^{2}=\frac{I_{k}(0)}{A_{k}(0)} ; s_{k}=\frac{L}{r_{k}} ; R_{k}=\frac{\bar{R}_{k}}{L} ; W_{k}(x)=\frac{\bar{W}_{k}\left(\bar{x}_{k}\right)}{L_{k}} ; \Psi_{k}(x)=\bar{\Psi}_{k}\left(\bar{x}_{k}\right) ; \\
a_{k}(x)=\frac{A_{k}\left(\bar{x}_{k}\right)}{A_{k}(0)} ; b_{k}(x)=\frac{I_{k}\left(\bar{x}_{k}\right)}{I_{k}(0)} ; v_{k}(x)=\frac{V_{k}\left(\bar{x}_{k}\right)}{l_{k} A_{k}(0)} ; \phi_{k}(x)=\frac{\Phi_{k}\left(\bar{x}_{k}\right)}{l_{k}^{2} A_{k}(0)} ; \\
N_{k+1}=\frac{\bar{F}_{k+1}}{E A_{k}(0)} ; N_{k}(x)=\frac{\bar{N}_{k}\left(\bar{x}_{k}\right)}{E A_{k}(0)} ; \quad Q_{k}(x)=\frac{\bar{Q}_{k}\left(\bar{x}_{k}\right)}{E A_{k}(0)} ; M_{k}(x)=\frac{L_{k}}{E I_{k}(0)} \bar{M}_{k}\left(\bar{x}_{k}\right) ;
\end{gathered}
$$


and $K_{W j}=\bar{K}_{W j} \frac{L}{E A_{1}(0)} ; K_{\Psi j}=\bar{K}_{\Psi j} \frac{L}{E I_{1}(0)} ;$ with $j=1$ or $j=d$.

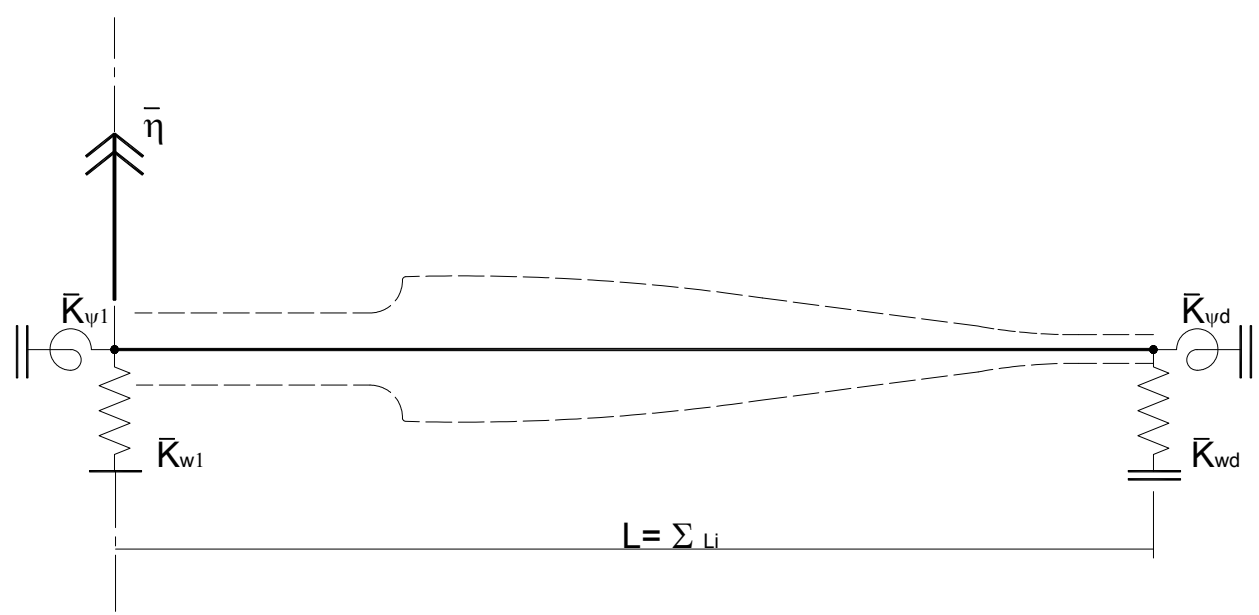

Fig. 3. Elastic restraints of the rotating beam

In each segment $k$ of the beam, $x$ varies between 0 and 1 .

The axial force, the shear force and the bending moment in the adimensional form become:

$$
\begin{gathered}
N_{k}(x)=\eta^{2} \frac{l_{k}^{2}}{s_{1}^{2}}\left(R_{k} v_{k}(1)+\phi_{k}(1)-R_{k} v_{k}(x)-\phi_{k}(x)\right)+N_{k+1} ; \text { with } s_{1}=\frac{L}{r_{1}} ; r_{1}^{2}=\frac{I_{1}(0)}{A_{1}(0)} \\
Q_{k}(x)=\left(N_{k}(x)+\frac{\kappa}{2(1+v)} a_{k}(x)\right) \frac{d W_{k}(x)}{d x}-\frac{\kappa}{2(1+v)} a_{k}(x) \Psi_{k}(x) \\
M_{k}(x)=b_{k}(x) \frac{d \Psi_{k}(x)}{d x}
\end{gathered}
$$

And the equations of motion in dimensionless form are:

$$
\begin{gathered}
\eta^{2} a_{k}(x)\left(R_{k}+x\right) \frac{d W_{k}(x)}{d x}-\frac{s_{1}^{2}}{l_{k}^{2}} N_{k}(x) \frac{d^{2} W_{k}(x)}{d x^{2}}-\frac{\kappa}{2(1+v)} \frac{s_{1}^{2}}{l_{k}^{2}} a_{k}(x)\left(\frac{d^{2} W_{k}(x)}{d x^{2}}-\frac{d \Psi_{k}(x)}{d x}\right)- \\
-\frac{\kappa}{2(1+v)} \frac{s_{1}^{2}}{l_{k}^{2}} \frac{d a_{k}(x)}{d x}\left(\frac{d W_{k}(x)}{d x}-\Psi_{k}(x)\right)=\Omega^{2} a_{k}(x) W_{k}(x) \\
-s_{1}^{2} \frac{\kappa}{2(1+v)} s_{k}^{2} a_{k}(x)\left(\frac{d W_{k}(x)}{d x}-\Psi_{k}(x)\right)-\frac{s_{1}^{2}}{l_{k}^{2}} b_{k}(x) \frac{d^{2} \Psi_{k}(x)}{d x^{2}}-\frac{s_{1}^{2}}{l_{k}^{2}} \frac{d b_{k}(x)}{d x} \frac{d \Psi_{k}(x)}{d x}- \\
-\eta^{2} b_{k}(x) \Psi_{k}(x)=\Omega^{2} b_{k}(x) \Psi_{k}(x)
\end{gathered}
$$


The equations (14), which satisfy continuity of displacement and rotation, can be expressed in dimensionless form as follows:

$$
l_{k} W_{k}(1)-l_{k+1} W_{k+1}(0)=0 ; \Psi_{k}(1)-\Psi_{k+1}(0)=0
$$

and the equations (15) of compatibility of the bending moment and the shear force, result in the following adimensional equations:

$$
\alpha_{k} Q_{k}(1)-\alpha_{k+1} Q_{k+1}(0)=0 ; \frac{\beta_{k}}{l_{k}} M_{k}(1)-\frac{\beta_{k+1}}{l_{k+1}} M_{k+1}(0)=0
$$

or

$$
\begin{gathered}
\left.\alpha_{k}\left[\left(N_{k}(x)+\frac{\kappa}{2(1+v)} a_{k}(x)\right) \frac{d W_{k}(x)}{d x}-\frac{\kappa}{2(1+v)} a_{k}(x) \Psi_{k}(x)\right]\right|_{x=1}- \\
-\left.\alpha_{k+1}\left[\left(N_{k+1}(x)+\frac{\kappa}{2(1+v)} a_{k+1}(x)\right) \frac{d W_{k+1}(x)}{d x}-\frac{\kappa}{2(1+v)} a_{k+1}(x) \Psi_{k+1}(x)\right]\right|_{x=0}=0 \\
\left.\frac{\beta_{k}}{l_{k}} b_{k}(x) \frac{d \Psi_{k}(x)}{d x}\right|_{x=1}-\left.\frac{\beta_{k+1}}{l_{k+1}} b_{k+1}(x) \frac{d \Psi_{k+1}(x)}{d x}\right|_{x=0}=0
\end{gathered}
$$

where $\alpha_{k}=\frac{A_{k}(0)}{A_{1}(0)} ; \beta_{k}=\frac{I_{k}(0)}{I_{1}(0)}$.

The boundary conditions at the end closest to the axis of rotation, segment $1, x=0$, are:

$$
\begin{gathered}
Q_{1}(0)-K_{W 1} l_{1} W_{1}(0)=0 ;\left.\left(N_{1}(0)+\frac{\kappa a_{1}(0)}{2(1+v)}\right) \frac{d W_{1}(x)}{d x}\right|_{x=0}-\frac{\kappa a_{1}(0) \Psi_{1}(0)}{2(1+v)}-K_{W 1} l_{1} W_{1}(0)=0 \\
M_{1}(0)-K_{\Psi 1} l_{1} \Psi_{1}(0)=0 ;\left.b_{1}(0) \frac{d \Psi_{1}(x)}{d x}\right|_{x=0}-K_{\Psi 1} l_{1} \Psi_{1}(0)=0
\end{gathered}
$$

and at the other end of the rotating beam, segment $d, x=1$, they are:

$$
\begin{gathered}
Q_{d}(1)-K_{W d} \frac{l_{d}}{\alpha_{d}} W_{d}(1)=0 ;\left.\left(N_{d}(1)+\frac{\kappa a_{d}(1)}{2(1+v)}\right) \frac{d W_{d}(x)}{d x}\right|_{x=1}-\frac{\kappa a_{d}(1)}{2(1+v)} \Psi_{d}(1)-\frac{K_{W d} l_{d}}{\alpha_{d}} W_{d}(1)=0 \\
M_{d}(1)-\frac{K_{\Psi d} l_{d}}{\beta_{d}} \Psi_{d}(1)=0 ;\left.b_{d}(1) \frac{d \Psi_{d}(x)}{d x}\right|_{x=1}-\frac{K_{\Psi d} l_{d}}{\beta_{d}} \Psi_{d}(1)=0
\end{gathered}
$$

where $N_{d}(1)$ is an outboard force at the end of the beam, farthest from the axis of rotation, that is equal to zero in the present study.

\section{Differential Quadrature Method, DQM}

In order to obtain the DQM analog equations from the governing equations of the rotating beam, the beam segment domain is discretized in a grid of $i$ points, using the Chebyshev Gauss - Lobato expression, (Shu, 2000). (See Fig. A.1 in Appendix A) 
Equations $(18,19,20)$ assumed the form:

$$
\begin{gathered}
N_{k}\left(x_{i}\right)=\eta^{2} \frac{l_{k}^{2}}{s_{1}^{2}}\left(R_{k} v_{k}(1)+\phi_{k}(1)-R_{k} v_{k}\left(x_{i}\right)-\phi_{k}\left(x_{i}\right)\right)+N_{k+1} \\
Q_{k}\left(x_{i}\right)=\left(N_{k}\left(x_{i}\right)+\frac{\kappa}{2(1+v)} a_{k}\left(x_{i}\right)\right) \sum_{j=1}^{n} A_{i j}^{(1)} W_{k j}-\frac{\kappa}{2(1+v)} a_{k}\left(x_{i}\right) \Psi_{k i} \\
M_{k}\left(x_{i}\right)=b_{k}\left(x_{i}\right) \sum_{j=1}^{n} A_{i j}^{(1)} \Psi_{k j}
\end{gathered}
$$

The equations of motion (21) and (22) become:

$$
\begin{aligned}
& \left(\eta^{2} a_{k}\left(x_{i}\right)\left(R_{k}+x_{i}\right)-\frac{\kappa}{2(1+v)} \frac{s_{1}^{2}}{l_{k}^{2}} \frac{d a_{k}\left(x_{i}\right)}{d x}\right) \sum_{j=1}^{n}\left(A_{i j}^{(1)}\right) W_{k j}- \\
& -\left(\frac{s_{1}^{2}}{l_{k}^{2}} N_{k}\left(x_{i}\right)+\frac{\kappa}{2(1+v)} \frac{s_{1}^{2}}{l_{k}^{2}} a_{k}\left(x_{i}\right)\right) \sum_{j=1}^{n}\left(A_{i j}^{(2)}\right) W_{k j}+\frac{\kappa}{2(1+v)} \frac{s_{1}^{2}}{l_{k}^{2}} a_{k}\left(x_{i}\right) \sum_{j=1}^{n} A_{i j}^{(1)} \Psi_{k j}+ \\
& +\frac{\kappa}{2(1+v)} \frac{s_{1}^{2}}{l_{k}^{2}} \frac{d a_{k}\left(x_{i}\right)}{d x} \Psi_{k i}=\Omega^{2} a_{k}\left(x_{i}\right) W_{k i} \\
& -\frac{\kappa}{2(1+v)} s_{1}^{2} s_{k}^{2} a_{k}\left(x_{i}\right) \sum_{j=1}^{n} A_{i j}^{(1)} W_{k j}-\frac{s_{1}^{2}}{l_{k}^{2}} b_{k}\left(x_{i}\right) \sum_{j=1}^{n} A_{i j}^{(2)} \Psi_{k j}+ \\
& +\left(\frac{\kappa}{2(1+v)} s_{1}^{2} s_{k}^{2} a_{k}\left(x_{i}\right)-\eta^{2} b_{k}\left(x_{i}\right)\right) \Psi_{k i}-\frac{s_{1}^{2}}{l_{k}^{2}} \frac{d b_{k}\left(x_{i}\right)}{d x} \sum_{j=1}^{n} A_{i j}^{(1)} \Psi_{k j}=\Omega^{2} b_{k}\left(x_{i}\right) \Psi_{k i}
\end{aligned}
$$

where the $A_{i j}^{(1)}$ and $A_{i j}^{(2)}$ are the weighting coefficients of linear algebraic equations. (See Appendix A.1 for more details).

Finally, the conditions (23) and (24) are replaced by:

$$
\begin{gathered}
l_{k} W_{k n}-l_{k+1} W_{(k+1) 1}=0 ; \Psi_{k n}-\Psi_{(k+1) 1}=0 ; \\
\alpha_{k}\left(\left(N_{k}(1)+\frac{\kappa}{2(1+v)} a_{k}(1)\right) \sum_{j=1}^{n} A_{n j}^{(1)} W_{k j}-\frac{\kappa}{2(1+v)} a_{k}(1) \Psi_{k n}\right) \\
-\alpha_{k+1}\left(\left(N_{k+1}(0)+\frac{\kappa}{2(1+v)} a_{k+1}(0)\right) \sum_{j=1}^{n} A_{1 j}^{(1)} W_{(k+1) j}-\frac{\kappa}{2(1+v)} a_{k+1}(0) \Psi_{k 1}\right)=0 ; \\
\frac{\alpha_{k}}{l_{k}} b_{k}(1) \sum_{j=1}^{n} A_{n j}^{(1)} \Psi_{k j}-\frac{\alpha_{k+1}}{l_{k+1}} b_{k+1}(0) \sum_{j=1}^{n} A_{1 j}^{(1)} \Psi_{(k+1) j}=0
\end{gathered}
$$

and the boundary conditions (25) and (26) replaced by: 


$$
\begin{gathered}
\left(N_{1}(0)+\frac{\kappa}{2(1+v)} a_{1}(0)\right) \sum_{j=1}^{n} A_{1 j}^{(1)} W_{1 j}-\frac{\kappa}{2(1+v)} a_{1}(0) \Psi_{11}-l_{1} K_{W 1} W_{11}=0 ; \\
K_{\Psi 1} \Psi_{11}-\frac{b_{1}(0)}{l_{1}} \sum_{j=1}^{n} A_{1 j}^{(1)} \Psi_{1 j}=0 \\
\left(N_{d}(1)+\frac{\kappa}{2(1+v)} a_{d}(1)\right) \sum_{j=1}^{n} A_{n j}^{(1)} W_{d j}-\frac{\kappa}{2(1+v)} a_{d}(1) \Psi_{d n}-l_{d} K_{W d} W_{d n}=0 ; \\
K_{\Psi n} \Psi_{d n}-\frac{b_{d}(1)}{l_{d}} \sum_{j=1}^{n} A_{n j}^{(1)} \Psi_{d j}=0
\end{gathered}
$$

The DQM linear equation system is used to determine the natural frequencies and mode shapes of the rotating beam.

The number of terms taken in the summations had been studied for many situations and the system has acceptable convergence by $n=21$ terms. (See Table 1 )

\section{Finite element method, MEF}

An independent set of results for the natural frequencies, was also obtained by a finite element code. (Bambill et al., 2010). The finite element model employed in the analysis has 3000 beam elements of two nodes in the longitudinal direction (Rossi, 2007). See Table 2. This number of elements was proved to be enough with a convergence analysis.

The beam model also takes into account the shear deformation (Timoshenko beam's theory) and the increase in bending stiffness induced by the centrifugal force.

The term $\rho I_{k}\left(\bar{x}_{k}\right) \bar{\eta}^{2} \bar{\Psi}_{k}\left(\bar{x}_{k}\right)$ of equation (13.b) was not included in the finite element formulation. Probably for this reason some small differences between both sets of numerical results (DQM and FEM) begin to appear when the rotational speed $\eta$ increases.

\section{Numerical results}

In the following examples some calculations were performed over elliptical cross sections. $(\kappa=0.886364)$. Without loss of generality, one may choose to keep constant width $e_{k}=e$ and vary the height $h_{k}(x)$ in each segment of the beam. The area and the second moment of area of the cross section of the beam will be $A_{k}(x)=\frac{\pi e h_{k}(x)}{4}, I_{k}(x)=\frac{\pi e h_{k}^{3}(x)}{64}$, and for this particular situation there are:

$$
a_{k}(x)=\frac{h_{k}(x)}{h_{k}(0)} ; b_{k}(x)=\left(\frac{h_{k}(x)}{h_{k}(0)}\right)^{3}
$$

The following formula is proposed to a quadratic variation of the height in each segment of beam:

$$
h_{k}(x)=c_{0 k}+c_{1 k} x+c_{2 k} x^{2}
$$


And the slope is the derivative of this function

$$
h_{k}^{\prime}(x)=\frac{d h_{k}(x)}{d x}=c_{1 k}+2 c_{2 k} x
$$

where $c_{0 k}, c_{1 k}$ and $c_{2 k}$ are constants, which are defined by the heights and slopes at both ends of each segment $k$. The heights and slopes at each end are identified with the subscript $A$ for $x=0: h_{A k} ; h_{A k}^{\prime}$ and with the subscript $B$ for $x=1: h_{B k} ; h_{B k}^{\prime}$.

If the segment of the beam shows a linear variation of height, $c_{2 k}=0$ and

$$
h_{A k}=c_{0 k} ; h_{B k}=c_{0 k}+c_{1 k} ; h_{A k}^{\prime}=h_{B k}^{\prime}=c_{1 k}
$$

As it can be seen in Table 1, the frequency coefficients calculated by the Differential Quadrature Method, DQM, using a summation with $n \geq 19(i=1,2,3, \ldots, n)$ points, show none significant improvement.

\begin{tabular}{|c|c|c|c|c|c|}
\hline $\mathrm{n}$ & $\Omega_{1}$ & $\Omega_{2}$ & $\Omega_{3}$ & $\Omega_{4}$ & $\Omega_{5}$ \\
\hline 5 & 15.6861 & 29.2939 & 49.1602 & 63.9792 & 112.610 \\
\hline 7 & 15.1981 & 28.9907 & 46.9070 & 64.9219 & 88.8670 \\
\hline 11 & 14.9057 & 29.5079 & 47.4960 & 64.7054 & 87.4079 \\
\hline 13 & 14.8340 & 29.6332 & 47.6579 & 64.7247 & 87.6724 \\
\hline 15 & 14.8291 & 29.6467 & 47.6811 & 64.7310 & 87.7047 \\
\hline 19 & 14.8295 & 29.6460 & 47.6816 & 64.7320 & 87.7080 \\
\hline 21 & 14.8296 & 29.6459 & 47.6815 & 64.7320 & 87.7080 \\
\hline
\end{tabular}

Table 1. Convergence analysis of the DQM, for a two-span rotating Timoshenko beam elastically restrained al both ends, with a quadratic variation of height.

The frequency coefficients in Table 1, correspond to a beam of two segments, rotating at speed $\eta=10$, whose characteristics are: elliptical cross section; $v=0.3 ; \kappa=0.886364$; $R_{1}=0 ; \quad l_{1} / L=l_{2} / L=1 / 2 ; \quad s_{1}=\sqrt{300} ; \quad h_{B 1} / h_{A 1}=1 / 2 ; \quad h_{B 1}^{\prime}=0 ; \quad h_{A 2} / h_{B 1}=1 / 2 ;$ $h_{B 2} / h_{A 2}=1 / 2 ; h_{A 2}^{\prime}=0 ; K_{W 1}=10 ; K_{\psi 1}=5 ; K_{W d}=0.1 ; K_{\psi d}=1$.

In Table 2 the values obtained for the natural frequency coefficients using the finite element method are presented for $\eta=\sqrt{\rho A_{0} / E I_{0}} L^{2} \bar{\eta}=0$ and $\eta=10$. The number of elements is increased from 10 to 3000 .

The model of the rotating beam of Table 2 has the following characteristics: one segment; rectangular cross section; $v=0.3 ; \kappa=10(1+v) /(12+11 v)=0.849673 ; R_{1}=0 ; \quad s_{1}=\sqrt{300}$; $h_{B} / h_{A}=1 / 4 ; h_{\mathrm{B}}^{\prime}=0 ; K_{W 1} \rightarrow \infty ; K_{\psi 1} \rightarrow \infty ; K_{W d}=0 ; K_{\psi d}=0$.

In the first examples it is assumed a perfect clamped condition at the axis of rotation, given by: $K_{W 1} \rightarrow \infty$ and $K_{\psi 1} \rightarrow \infty$. (Tables 3,4 and 5).

Table 3 presents the effect of the rotational speed parameter $\eta$ on the natural frequency coefficients of a rotating cantilever beam of one segment, $\left(K_{W 1} \rightarrow \infty ; K_{\psi 1} \rightarrow \infty ; K_{W d}=0\right.$; $\left.K_{\psi d}=0\right)$. The results correspond to a linear variation of height and a comparison is made with 
(Barnejee, 2006) when Banerjee's parameter is $n=1$. As it can be observed the agreement is excellent.

\begin{tabular}{|c|c|c|c|c|c|}
\hline \multicolumn{7}{|c|}{$\eta=0$} \\
\hline Number of elements & $\Omega_{1}$ & $\Omega_{2}$ & $\Omega_{3}$ & $\Omega_{4}$ & $\Omega_{5}$ \\
\hline 10 & 3.38628165 & 11.7689336 & 26.5951854 & 46.6658427 & 71.0448001 \\
\hline 100 & 3.37398143 & 11.7248502 & 26.4438604 & 46.1408176 & 69.5136708 \\
\hline 1000 & 3.37385398 & 11.7243988 & 26.4423706 & 46.1357196 & 69.4986357 \\
\hline 2000 & 3.37385302 & 11.7243954 & 26.4423593 & 46.1356810 & 69.4985219 \\
\hline 3000 & 3.37385284 & 11.7243946 & 26.4423572 & 46.1356739 & 69.4985008 \\
\hline \multicolumn{7}{|c|}{$\eta=10$} \\
\hline 10 & 11.6074237 & 25.8805102 & 44.0407905 & 66.3753084 & 92.6859627 \\
\hline 100 & 11.6098042 & 25.7094320 & 43.5638284 & 65.4674874 & 90.8491237 \\
\hline 1000 & 11.6098077 & 25.7074626 & 43.5585908 & 65.4579769 & 90.8301746 \\
\hline 2000 & 11.6098078 & 25.7074476 & 43.5585511 & 65.4579049 & 90.8300310 \\
\hline 3000 & 11.6098078 & 25.7074448 & 43.5585437 & 65.4578915 & 90.8300044 \\
\hline
\end{tabular}

Table 2. Convergence analysis of the frequency coefficients $\Omega_{i}=\sqrt{\rho A_{0} / E I_{0}} L^{2} \omega_{i}$ using MEF.

\begin{tabular}{|c|c|c|c|c|c|c|}
\hline$\eta$ & & $\Omega_{1}$ & $\Omega_{2}$ & $\Omega_{3}$ & $\Omega_{4}$ & $\Omega_{5}$ \\
\hline \multirow{2}{*}{0} & $\overline{\mathrm{DQM}}$ & 3.82377 & 18.3171 & 47.2638 & 90.4468 & 147.992 \\
\hline & (Barnejee,2006) & 3.82379 & 18.3173 & 47.2648 & 90.4505 & 148.002 \\
\hline \multirow{2}{*}{2} & DQM & 4.43680 & 18.9365 & 47.8706 & 91.0589 & 148.609 \\
\hline & (Barnejee,2006) & 4.43680 & 18.9366 & 47.8717 & 91.0625 & 148.619 \\
\hline \multirow{2}{*}{4} & DQM & 5.87874 & 20.6850 & 49.6446 & 92.8693 & 150.444 \\
\hline & (Barnejee, 2006) & 5.87877 & 20.6851 & 49.6456 & 92.8730 & 150.454 \\
\hline \multirow{2}{*}{6} & DQMM & 7.65512 & 23.3091 & 52.4622 & 95.8054 & 153.450 \\
\hline & (Barnejee,2006) & 7.65514 & 23.3093 & 52.4632 & 95.8090 & 153.460 \\
\hline \multirow{2}{*}{8} & DQM & 9.55392 & 26.5435 & 56.1584 & 99.7601 & 157.555 \\
\hline & (Barnejee,2006) & 9.55396 & 26.5437 & 56.1595 & 99.7638 & 157.564 \\
\hline \multirow{2}{*}{10} & DQM & 11.5015 & 30.1825 & 60.5628 & 104.608 & 162.668 \\
\hline & (Barnejee,2006) & 11.5015 & 30.1827 & 60.5639 & 104.612 & 162.677 \\
\hline
\end{tabular}

Table 3. Frequency coefficients $\Omega_{i}=\sqrt{\rho A_{1}(0) / E I_{1}(0)} L^{2} \omega_{i}$ for a one-span beam, $l_{1} / L=1$; $s_{1}=\sqrt{1000} ; h_{B} / h_{A}=1 / 2 ; K_{W 1} \rightarrow \infty ; K_{\psi 1} \rightarrow \infty ; K_{W d}=0 ; K_{\psi d}=0$.

All the calculations performed for the following Tables and Graphics used $R_{1}=0$; and $v=0.30 ; \kappa=0.886364$ (elliptical cross section).

The DQM results are determined using $n=21$ in each segment of the beam, and the MEF results were obtained with 3000 elements.

The beam considered in Table 4 has one segment and is elastically restrained at its outer end. The parameter of rotation speed $\eta$ is taken equal to 10 . The Table presents the frequency coefficients for the first five mode shapes which correspond to different sets of elastically boundary conditions given by the spring constant parameters $K_{W d}$ and $K_{\psi d}$. The other details of the beam are specified in the legend of the table. 
The beam model considered in Table 5 has two segments of equal length and similar conditions and parameters as Table 4.

\begin{tabular}{|c|c|c|c|c|c|c|c|}
\hline$K_{\psi d}$ & $K_{W d}$ & Method & $\Omega_{1}$ & $\Omega_{2}$ & $\Omega_{3}$ & $\Omega_{4}$ & $\Omega_{5}$ \\
\hline \multirow{10}{*}{0} & & DQM & 11.2148 & 27.6174 & 50.0089 & 77.5866 & 108.472 \\
\hline & & FEM & 11.2375 & 27.6743 & 50.0711 & 77.6432 & 108.523 \\
\hline & \multirow{2}{*}{0.1} & $\overline{\mathrm{DQ}} \overline{\mathrm{M}}$ & 15.4254 & 32.5178 & $\overline{5} \overline{2} . \overline{8516}$ & 79.0733 & 109.357 \\
\hline & & FEM & 15.4438 & 32.5548 & 52.9087 & 79.1298 & 109.408 \\
\hline & \multirow{2}{*}{1} & $\overline{\mathrm{DQM}}$ & 18.0157 & 40.3494 & 65.6538 & 92.2848 & 119.836 \\
\hline & & FEM & 18.0465 & 40.3841 & 65.6882 & 92.3208 & 119.877 \\
\hline & \multirow{2}{*}{10} & $\overline{\mathrm{D}} \overline{\mathrm{M}}$ & 18.3978 & 41.6361 & 69.0216 & 99.4111 & 131.859 \\
\hline & & FEM & 18.4315 & 41.6757 & 69.0611 & 99.4484 & 131.893 \\
\hline & \multirow{2}{*}{$\rightarrow \infty$} & $\overline{\mathrm{D}} \overline{\mathrm{M}}$ & $18.441 \overline{7}$ & 41.7750 & $69 . \overline{3474}$ & 100.033 & 132.894 \\
\hline & & $\widehat{F E M}$ & 18.4757 & 41.8151 & 69.3878 & 100.071 & 132.929 \\
\hline \multirow{10}{*}{1} & & DQM & 11.3941 & 29.3678 & 53.0174 & 81.0192 & 112.024 \\
\hline & & FEM & 11.4148 & 29.4104 & 53.0660 & 81.0662 & 112.068 \\
\hline & \multirow{2}{*}{0.1} & $\overline{\mathrm{D}} \overline{\mathrm{Q}} \overline{\mathrm{M}}$ & 15.6233 & 32.8965 & 55.0307 & 82.2247 & 112.825 \\
\hline & & FEM & 15.6400 & 32.9308 & 55.0763 & 82.2710 & 112.868 \\
\hline & \multirow[b]{2}{*}{1} & $\overline{\mathrm{DQ}} \overline{\mathrm{M}} \overline{\mathrm{M}}$ & 19.2962 & 41.3980 & 65.9339 & 92.3365 & 120.822 \\
\hline & & FEM & 19.3219 & 41.4295 & 65.9674 & 92.3723 & 120.859 \\
\hline & \multirow{2}{*}{10} & $\overline{\mathrm{DQ}} \overline{\mathrm{M}}$ & 19.9179 & 43.3987 & 70.5662 & 100.622 & 132.723 \\
\hline & & FEM & 19.9463 & 43.4345 & 70.6034 & 100.658 & 132.756 \\
\hline & \multirow{2}{*}{$\rightarrow \infty$} & $\overline{\mathrm{D}} \overline{\mathrm{M}}$ & 19.9899 & 43.6199 & 71.0558 & 101.509 & 134.136 \\
\hline & & FEM & 20.0187 & 43.6562 & 71.0937 & 101.546 & 134.170 \\
\hline \multirow{10}{*}{10} & \multirow{2}{*}{0} & DQM & 11.4913 & 30.3954 & 55.1815 & 84.0260 & 115.628 \\
\hline & & FEM & 11.5115 & 30.4328 & 55.2229 & 84.0663 & 115.665 \\
\hline & \multirow{2}{*}{0.1} & $\overline{\mathrm{Q}} \overline{\mathrm{M}}$ & 15.7621 & 33.1503 & 56.5688 & 84.8630 & 116.210 \\
\hline & & FEM & 15.7780 & 33.1835 & 56.6092 & 84.9031 & 116.247 \\
\hline & \multirow{2}{*}{1} & $\overline{\mathrm{D}} \overline{\mathrm{Q}} \overline{\mathrm{M}}$ & 20.4765 & $4 \overline{2} .5961$ & $\overline{6} \overline{6} . \overline{2} \overline{6} \overline{5}$ & 92.3899 & 121.730 \\
\hline & & FEM & 20.4994 & 42.6248 & 66.2963 & 92.4255 & 121.765 \\
\hline & \multirow{2}{*}{10} & $\overline{\mathrm{D}} \overline{\mathrm{M}}$ & 21.3539 & 45.5548 & $\overline{7} 2.8197$ & 102.560 & 134.141 \\
\hline & & FEM & 21.3795 & 45.5875 & 72.8543 & 102.594 & 134.174 \\
\hline & \multirow{2}{*}{$\rightarrow \infty$} & $\overline{\mathrm{Q}} \overline{\mathrm{M}}$ & 21.4553 & 45.8807 & 73.5609 & 103.912 & 136.261 \\
\hline & & FEM & 21.4813 & 45.9139 & 73.5962 & 103.947 & 136.294 \\
\hline \multirow{10}{*}{$\rightarrow \infty$} & & DQM & 11.5091 & 30.5860 & 55.6105 & 84.6706 & 116.454 \\
\hline & & FEM & 11.5291 & 30.6228 & 55.6510 & 84.7101 & 116.491 \\
\hline & \multirow{2}{*}{0.1} & $\overline{\mathrm{D}} \overline{\mathrm{Q}} \overline{\mathrm{M}}$ & 15.7905 & 33.2010 & $\overline{5} \overline{6} . \overline{8768}$ & 85.4233 & $\overline{11} \overline{6} . \overline{975}$ \\
\hline & & FEM & 15.8064 & 33.2340 & 56.9165 & 85.4625 & 117.012 \\
\hline & \multirow[t]{2}{*}{1} & $\overline{\mathrm{D}} \overline{\mathrm{M}}$ & 20.7557 & 42.9193 & 66.3549 & 92.4035 & 121.943 \\
\hline & & FEM & 20.7782 & 42.9475 & 66.3875 & 92.4392 & 121.978 \\
\hline & \multirow{2}{*}{10} & $\overline{\mathrm{D}} \overline{\mathrm{Q}} \overline{\mathrm{M}}$ & 21.6961 & 46.1510 & $\overline{7} \overline{5} \overline{5} \overline{285}$ & 103.223 & $\overline{134} . \overline{643}$ \\
\hline & & FEM & 21.7214 & 46.1832 & 73.5626 & 103.257 & 134.675 \\
\hline & \multirow{2}{*}{$\rightarrow \infty$} & $\overline{\mathrm{D}} \overline{\mathrm{M}}$ & 21.8045 & 46.5039 & 74.3452 & 104.735 & 137.026 \\
\hline & & FEM & 21.8302 & 46.5368 & 74.3801 & 104.769 & 137.059 \\
\hline
\end{tabular}

Table 4. First natural frequencies $\Omega_{i}=\sqrt{\rho A_{1}(0) / E I_{1}(0)} L^{2} \omega_{i}$ for a one-span rotating Timoshenko beam, with elliptical cross section and quadratic height variation along the axis. $v=0.3 ; s_{1}=\sqrt{300} ; h_{B} / h_{A}=1 / 2 ; h_{B}^{\prime}=0 ; K_{W 1} \rightarrow \infty ; K_{\psi 1} \rightarrow \infty ; \eta=10$. 


\begin{tabular}{|c|c|c|c|c|c|c|c|}
\hline$K_{\psi d}$ & $K_{W d}$ & Method & $\Omega_{1}$ & $\Omega_{2}$ & $\Omega_{3}$ & $\Omega_{4}$ & $\Omega_{5}$ \\
\hline \multirow{10}{*}{0} & & DQM & 11.8651 & 24.5717 & 40.8347 & 59.8775 & 81.1573 \\
\hline & & FEM & 11.8796 & 24.5914 & 40.8559 & 59.9110 & 81.1826 \\
\hline & \multirow{2}{*}{0.1} & DQM & 15.2667 & 30.3903 & 49.8409 & 67.9228 & 90.5546 \\
\hline & & FEM & 15.2858 & 30.4064 & 49.8638 & 67.9498 & 90.5743 \\
\hline & \multirow{2}{*}{1} & $\overline{\mathrm{DQM}}$ & 15.6938 & 31.4585 & 52.2093 & 72.0062 & $\overline{98.7038}$ \\
\hline & & FEM & 15.7140 & 31.4754 & 52.2362 & 72.0330 & 98.7265 \\
\hline & & $\mathrm{DQM}$ & 15.7412 & 31.5756 & 52.4458 & 72.4214 & 99.4803 \\
\hline & & FEM & 15.7616 & 31.5927 & 52.4732 & 72.4482 & 99.5038 \\
\hline & \multirow{2}{*}{$\rightarrow \infty$} & $\overline{\mathrm{DQM}}$ & 15.7466 & 31.5887 & 52.4718 & 72.4669 & 99.5627 \\
\hline & & FEM & 15.7669 & 31.6059 & 52.4993 & 72.4937 & 99.5862 \\
\hline \multirow{10}{*}{1} & & DQM & 11.9142 & 25.1342 & 42.8878 & 62.4877 & 85.6040 \\
\hline & & FEM & 11.9288 & 25.1532 & 42.9079 & 62.5196 & 85.6258 \\
\hline & \multirow{2}{*}{0.1} & $\overline{\mathrm{DQ}} \overline{\mathrm{M}}$ & 16.2121 & 31.6526 & 50.5459 & 67.9979 & 90.8436 \\
\hline & & FEM & 16.2314 & 31.6672 & 50.5682 & 68.0245 & 90.8635 \\
\hline & \multirow[b]{2}{*}{1} & $\mathrm{DQM}$ & 16.9952 & 33.8476 & 55.0090 & 75.3283 & 102.166 \\
\hline & & FEM & 17.0160 & 33.8634 & 55.0372 & 75.3520 & 102.190 \\
\hline & \multirow[b]{2}{*}{10} & $\overline{\mathrm{DQM}}$ & 17.0842 & 34.0961 & 55.4704 & 76.2542 & 103.723 \\
\hline & & FEM & 17.1052 & 34.1120 & 55.4993 & 76.2779 & 103.748 \\
\hline & \multirow{2}{*}{$\rightarrow \infty$} & DQM & 17.0942 & 34.1238 & $\overline{5} \overline{5} . \overline{5} 20 \overline{5}$ & 76.3541 & 103.882 \\
\hline & & FEM & 17.1152 & 34.1398 & 55.5496 & 76.3778 & 103.907 \\
\hline \multirow{10}{*}{10} & & DQM & 11.9157 & 25.1505 & 42.9498 & 62.5733 & 85.7690 \\
\hline & & FEM & 11.9302 & 25.1695 & 42.9699 & 62.6051 & 85.7907 \\
\hline & \multirow{2}{*}{0.1} & $\mathrm{DQM}$ & 16.2528 & 31.7152 & 50.5831 & 68.0018 & 90.8571 \\
\hline & & FEM & 16.2721 & 31.7297 & 50.6053 & 68.0283 & 90.8770 \\
\hline & \multirow[t]{2}{*}{1} & $\mathrm{DQM}$ & 17.0528 & 33.9729 & 55.1728 & 75.5622 & 102.430 \\
\hline & & FEM & 17.0737 & 33.9886 & $\underline{5} 5.2011$ & 75.5857 & 102.453 \\
\hline & \multirow{2}{*}{10} & $\overline{\mathrm{D}} \overline{\mathrm{Q}} \overline{\mathrm{M}}$ & 17.1437 & 34.2281 & 55.6450 & 76.5200 & 104.034 \\
\hline & & FEM & 17.1648 & 34.2440 & 55.6741 & 76.5435 & 104.059 \\
\hline & \multirow{2}{*}{$\rightarrow \infty$} & DQM & 17.1539 & 34.2566 & 55.6962 & 76.6231 & 104.197 \\
\hline & & FEM & 17.1750 & 34.2725 & 55.7255 & 76.6466 & 104.222 \\
\hline \multirow{10}{*}{$\rightarrow \infty$} & & $\overline{\mathrm{DQM}}$ & 11.9158 & 25.1524 & 42.9569 & 62.5831 & 85.7880 \\
\hline & & FEM & 11.9304 & 25.1713 & 42.9770 & 62.6149 & 85.8097 \\
\hline & \multirow{2}{*}{0.1} & DQM & 16.2575 & 31.7225 & 50.5875 & 68.0023 & 90.8587 \\
\hline & & FEM & 16.2768 & 31.7370 & 50.6097 & 68.0288 & 90.8786 \\
\hline & & $\overline{\mathrm{DQ}} \overline{\mathrm{M}}$ & 17.0595 & 33.9876 & 55.1921 & 75.5901 & 102.461 \\
\hline & & FEM & 17.0804 & 34.0033 & 55.2205 & 75.6136 & 102.485 \\
\hline & \multirow{2}{*}{10} & DQM & 17.1506 & 34.2436 & 55.6656 & 76.5517 & 104.071 \\
\hline & & FEM & 17.1717 & 34.2595 & 55.6947 & 76.5752 & 104.096 \\
\hline & \multirow{2}{*}{$\rightarrow \infty$} & DQM & 17.1609 & 34.2722 & 55.7169 & 76.6551 & 104.234 \\
\hline & & FEM & 17.1820 & 34.2881 & 55.7461 & 76.6786 & 104.259 \\
\hline
\end{tabular}

Table 5. First natural frequencies $\Omega_{i}=\sqrt{\rho A_{1}(0) / E I_{1}(0)} L^{2} \omega_{i}$ for a two-span elastically restrained rotating Timoshenko beam, with elliptical cross section and quadratic height variation along the axis. $l_{1} / L=1 / 2 l_{2} / L=1 / 2, h_{B 1} / h_{A 1}=1 / 2, h_{B 1}^{\prime}=0$, $h_{A 2} / h_{B 1}=1 / 2, h_{B 2} / h_{A 2}=1 / 2, h_{A 2}^{\prime}=0, K_{W 1} \rightarrow \infty K_{\psi 1} \rightarrow \infty, \eta=10$. 
Next Tables, 6 to 10, correspond to beams of two segments, elastically restrained at both ends and any particular details are expressed in each legend.

\begin{tabular}{|c|c|c|c|c|c|c|c|}
\hline$K_{\psi d}$ & $K_{W d}$ & Method & $\Omega_{1}$ & $\Omega_{2}$ & $\Omega_{3}$ & $\Omega_{4}$ & $\Omega_{5}$ \\
\hline \multirow{10}{*}{0} & \multirow{2}{*}{0} & DQM & 9.98841 & 21.2706 & 37.3110 & 54.9224 & 77.7336 \\
\hline & & FEM & 10.0246 & 21.3074 & 37.3506 & 54.9699 & 77.7658 \\
\hline & \multirow{2}{*}{0.1} & $\overline{\mathrm{D}} \overline{\mathrm{Q}} \overline{\mathrm{M}}$ & 12.4181 & 26.7466 & $\overline{4} 5 . \overline{4} 389$ & 63.7717 & $8 \overline{8} . \overline{4} 9 \overline{4}$ \\
\hline & & FEM & 12.4641 & 26.7810 & 45.4827 & 63.8054 & 87.5227 \\
\hline & \multirow{2}{*}{1} & $\overline{\mathrm{DQ}} \overline{\mathrm{Q}} \overline{\mathrm{M}}$ & 12.7051 & 27.6834 & $\overline{4} \overline{7} . \overline{3} 15 \overline{4}$ & 67.8701 & $\overline{9} \overline{9} \overline{4} \overline{48}$ \\
\hline & & FEM & 12.7526 & 27.7193 & 47.3634 & 67.9035 & 94.9788 \\
\hline & \multirow{2}{*}{10} & $\overline{\mathrm{D}} \overline{\mathrm{Q}} \overline{\mathrm{M}}$ & 12.7370 & $2 \overline{7} . \overline{7869}$ & $\overline{4} \overline{7} . \overline{506 \overline{5}}$ & 68.2901 & $\overline{9} \overline{5} . \overline{6} 98$ \\
\hline & & FEM & 12.7847 & 27.8230 & 47.5548 & 68.3236 & 95.6747 \\
\hline & \multirow{2}{*}{$\rightarrow \infty$} & $\overline{\mathrm{D}} \overline{\mathrm{Q}} \overline{\mathrm{M}}$ & $12 . \overline{7} 40 \overline{6}$ & 27.7985 & $\overline{4} \overline{7} . \overline{5} 2 \overline{\overline{6}}$ & 68.3362 & $\overline{9} \overline{\bar{y}} \overline{\overline{7}} \overline{137}$ \\
\hline & & FEM & 12.7883 & 27.8347 & 47.5760 & 68.3697 & 95.7487 \\
\hline \multirow{10}{*}{1} & \multirow{2}{*}{0} & DQM & 10.0086 & 21.6505 & 39.0007 & 57.4853 & 82.1930 \\
\hline & & FEM & 10.0451 & 21.6876 & 39.0412 & 57.5291 & 82.2227 \\
\hline & \multirow{2}{*}{0.1} & $\overline{\mathrm{DQ}} \overline{\mathrm{M}}$ & 13.1047 & 28.0775 & $\overline{4} 6 . \overline{2626}$ & 63.9615 & 87.6768 \\
\hline & & FEM & 13.1521 & 28.1101 & 46.3049 & 63.9941 & 87.7052 \\
\hline & \multirow[b]{2}{*}{1} & $\overline{\mathrm{DQ}} \overline{\mathrm{M}} \overline{\mathrm{M}}$ & 13.6220 & 29.9765 & $\overline{4} \overline{9} . \overline{8} 84 \overline{7}$ & $71.508 \overline{4}$ & $\overline{98} . \overline{2} \overline{67}$ \\
\hline & & FEM & 13.6718 & 30.0120 & 49.9325 & 71.5384 & 98.3117 \\
\hline & \multirow{2}{*}{10} & $\overline{\mathrm{DQ}} \overline{\mathrm{Q}} \overline{\mathrm{M}}$ & 13.6812 & 30.1918 & 50.2664 & 72.4302 & $\overline{99} . \overline{6} \overline{6} 46$ \\
\hline & & FEM & 13.7312 & 30.2278 & 50.3147 & 72.4604 & 99.7012 \\
\hline & \multirow{2}{*}{$\rightarrow \infty$} & $\overline{\mathrm{D} Q \bar{M}} \overline{\mathrm{M}}$ & 13.6879 & 30.2159 & $50 . \overline{3082}$ & 72.5299 & $\overline{9} \overline{8} . \overline{8074}$ \\
\hline & & FEM & 13.7379 & 30.2519 & 50.3566 & 72.5601 & 99.8441 \\
\hline \multirow{10}{*}{10} & & $\overline{\mathrm{DQM}}$ & 10.0092 & 21.6615 & 39.0505 & 57.5689 & 82.3537 \\
\hline & & FEM & 10.0457 & 21.6987 & 39.0910 & 57.6127 & 82.3835 \\
\hline & \multirow[b]{2}{*}{0.1} & $\overline{\mathrm{DQ}} \overline{\mathrm{M}}$ & 13.1336 & 28.1416 & $\overline{4} \overline{6} . \overline{3059}$ & 63.9714 & $8 \overline{7} . \overline{6854}$ \\
\hline & & FEM & 13.1811 & 28.1741 & 46.3481 & 64.0039 & 87.7138 \\
\hline & & $\overline{\mathrm{DQ}} \overline{\mathrm{M}}$ & 13.6618 & 30.0922 & 50.0321 & 71.7561 & $98 . \overline{5} 256$ \\
\hline & & FEM & 13.7116 & 30.1278 & 50.0799 & 71.7860 & 98.5607 \\
\hline & \multirow{2}{*}{10} & $\overline{\mathrm{D}} \overline{\mathrm{Q}} \overline{\mathrm{M}}$ & 13.7222 & 30.3131 & $50 . \overline{4} 233$ & 72.7081 & $\overline{99.9556}$ \\
\hline & & FEM & 13.7723 & 30.3491 & 50.4716 & 72.7381 & 99.9923 \\
\hline & \multirow{2}{*}{$\rightarrow \infty$} & $\overline{\mathrm{D}} \overline{\mathrm{Q}} \overline{\mathrm{M}}$ & 13.7290 & 30.3379 & $\overline{5} 0 . \overline{4} 6 \overline{1}$ & 72.8107 & $\overline{1} 0 . \overline{102}$ \\
\hline & & FEM & 13.7792 & 30.3739 & 50.5145 & 72.8408 & 100.139 \\
\hline \multirow{10}{*}{$\rightarrow \infty$} & & $\overline{D Q M}$ & 10.0093 & 21.6628 & 39.0562 & 57.5786 & 82.3722 \\
\hline & & FEM & 10.0458 & 21.6999 & 39.0968 & 57.6224 & 82.4020 \\
\hline & \multirow{2}{*}{0.1} & $\overline{\mathrm{D}} \overline{\mathrm{Q}} \overline{\mathrm{M}}$ & 13.1369 & $28.1491^{-}$ & $\overline{4} \overline{6} . \overline{3} \overline{110}$ & $\overline{63.972 \overline{6}}$ & $\overline{87} . \overline{6} \overline{8} 64$ \\
\hline & & FEM & 13.1844 & 28.1817 & 46.3532 & 64.0051 & 87.7149 \\
\hline & & $\overline{\mathrm{D} Q \bar{M}} \overline{\mathrm{M}}$ & 13.6664 & 30.1057 & 50.0495 & $71.78 \overline{5} \overline{6}$ & $\overline{98 . \overline{5} 555}$ \\
\hline & & FEM & 13.7163 & 30.1414 & 50.0973 & 71.8155 & 98.5906 \\
\hline & \multirow{2}{*}{10} & $\overline{\mathrm{DQ}} \overline{\mathrm{M}} \overline{\mathrm{C}}$ & 13.7270 & 30.3273 & $50 . \overline{4} 18$ & 72.7411 & 99.9903 \\
\hline & & FEM & 13.7771 & 30.3634 & 50.4901 & 72.7711 & 100.027 \\
\hline & \multirow{2}{*}{$\rightarrow \infty$} & $\overline{\mathrm{DQ}} \overline{\mathrm{Q}} \overline{\mathrm{M}}$ & 13.7338 & 30.3521 & $50 . \overline{4} 847$ & 72.8441 & 100.137 \\
\hline & & FEM & 13.7840 & 30.3882 & 50.5331 & 72.8741 & 100.174 \\
\hline
\end{tabular}

Table 6. First natural frequencies $\Omega_{i}=\sqrt{\rho A_{1}(0) / E I_{1}(0)} L^{2} \omega_{i}$ for a two-span elastically restrained rotating Timoshenko beam, with elliptical cross section and quadratic height variation along the axis. $l_{1} / L=1 / 2 l_{2} / L=1 / 2, h_{B 1} / h_{A 1}=1 / 2, h_{B 1}^{\prime}=0$, $h_{A 2} / h_{B 1}=1 / 2, h_{B 2} / h_{A 2}=1 / 2, h_{A 2}^{\prime}=0, K_{W 1} \rightarrow \infty, K_{\psi 1}=0.1, \eta=10$. 


\begin{tabular}{|c|c|c|c|c|c|c|c|}
\hline$K_{\psi d}$ & $K_{W d}$ & Method & $\Omega_{1}$ & $\Omega_{2}$ & $\Omega_{3}$ & $\Omega_{4}$ & $\Omega_{5}$ \\
\hline \multirow{10}{*}{0} & & DQM & 11.3734 & 23.4059 & 39.2570 & 56.9363 & 78.4787 \\
\hline & & FEM & 11.3904 & 23.4273 & 39.2820 & 56.9721 & 78.5041 \\
\hline & \multirow{2}{*}{0.1} & $\overline{\mathrm{Q}} \overline{\mathrm{M}}$ & 14.4551 & 28.9705 & 47.6260 & 65.1400 & 87.8678 \\
\hline & & FEM & 14.4773 & 28.9890 & 47.6542 & 65.1664 & 87.8894 \\
\hline & \multirow[b]{2}{*}{1} & $\overline{\mathrm{DQM}}$ & 14.8320 & 29.9641 & 49.6507 & 69.1005 & $\overline{95} . \overline{0494}$ \\
\hline & & FEM & 14.8553 & 29.9837 & 49.6827 & 69.1264 & 95.0757 \\
\hline & & DQM & 14.8738 & 30.0733 & 49.8536 & 69.5057 & 95.7100 \\
\hline & & FEM & 14.8972 & 30.0931 & 49.8861 & 69.5316 & 95.7371 \\
\hline & \multirow{2}{*}{$\rightarrow \infty$} & $\overline{\mathrm{DQ}} \overline{\mathrm{M}}$ & 14.8785 & 30.0856 & $\overline{49.8761}$ & 69.5501 & $\overline{95.7801}$ \\
\hline & & FEM & 14.9019 & 30.1054 & 49.9086 & 69.5761 & 95.8073 \\
\hline \multirow{10}{*}{1} & & DQM & 11.4126 & 23.8883 & 41.1048 & 59.4030 & 82.7965 \\
\hline & & FEM & 11.4297 & 23.9092 & 41.1294 & 59.4361 & 82.8192 \\
\hline & \multirow{2}{*}{0.1} & $\overline{\mathrm{DQM}}$ & 15.3087 & 30.2367 & 48.3683 & 65.2804 & 88.0547 \\
\hline & & FEM & 15.3312 & 30.2539 & 48.3957 & 65.3061 & 88.0766 \\
\hline & & DQM & 15.9925 & 32.2677 & 52.2109 & 72.5363 & 98.2033 \\
\hline & & FEM & 16.0166 & 32.2865 & 52.2436 & 72.5592 & 98.2306 \\
\hline & \multirow{2}{*}{10} & $\overline{\mathrm{DQ}} \overline{\mathrm{M}}$ & 16.0702 & 32.4974 & 52.6080 & 73.4315 & $\overline{99.5067}$ \\
\hline & & FEM & 16.0945 & 32.5165 & 52.6413 & 73.4545 & 99.5353 \\
\hline & \multirow{2}{*}{$\rightarrow \infty$} & $\mathrm{DQM}$ & 16.0790 & 32.5231 & 52.6513 & 73.5282 & 99.6404 \\
\hline & & FEM & 16.1032 & 32.5422 & 52.6847 & 73.5513 & 99.6691 \\
\hline \multirow{10}{*}{10} & & DQM & 11.4138 & 23.9023 & 41.1598 & 59.4837 & 82.9526 \\
\hline & & FEM & 11.4309 & 23.9232 & 41.1844 & 59.5168 & 82.9753 \\
\hline & \multirow{2}{*}{0.1} & $\mathrm{DQM}$ & 15.3450 & 30.2988 & 48.4074 & 65.2877 & 88.0636 \\
\hline & & FEM & 15.3676 & 30.3159 & 48.4348 & 65.3134 & 88.0854 \\
\hline & & $\overline{\mathrm{DQM}}$ & 16.0434 & 32.3866 & 52.3585 & 72.7730 & 98.4379 \\
\hline & & FEM & 16.0676 & 32.4055 & 52.3914 & 72.7958 & 98.4653 \\
\hline & \multirow{2}{*}{10} & $\overline{\mathrm{D}} \overline{\mathrm{M}}$ & 16.1228 & 32.6224 & 52.7651 & 73.6979 & 99.7796 \\
\hline & & FEM & 16.1471 & 32.6415 & 52.7985 & 73.7208 & 99.8083 \\
\hline & \multirow{2}{*}{$\rightarrow \infty$} & $\overline{D Q M}$ & 16.1317 & 32.6487 & 52.8094 & 73.7975 & 99.9164 \\
\hline & & FEM & 16.1560 & 32.6679 & 52.8428 & 73.8204 & 99.9453 \\
\hline \multirow{10}{*}{$\rightarrow \infty$} & & DQM & 11.4139 & 23.9039 & 41.1661 & 59.4929 & 82.9706 \\
\hline & & FEM & 11.4310 & 23.9248 & 41.1908 & 59.5260 & 82.9933 \\
\hline & \multirow{2}{*}{0.1} & DQM & 15.3493 & 30.3061 & 48.4120 & 65.2886 & 88.0646 \\
\hline & & FEM & 15.3718 & 30.3232 & 48.4394 & 65.3142 & 88.0865 \\
\hline & & $\overline{\mathrm{DQ}} \overline{\mathrm{M}}$ & 16.0493 & 32.4005 & 52.3759 & 72.8012 & $\overline{98.4661}$ \\
\hline & & FEM & 16.0735 & 32.4194 & 52.4088 & 72.8240 & 98.4934 \\
\hline & \multirow[t]{2}{*}{10} & DQM & 16.1289 & 32.6371 & 52.7836 & 73.7295 & 99.8122 \\
\hline & & FEM & 16.1532 & 32.6562 & 52.8170 & 73.7524 & 99.8409 \\
\hline & \multirow{2}{*}{$\rightarrow \infty$} & $\mathrm{DQM}$ & 16.1378 & 32.6635 & 52.8280 & 73.8295 & 99.9493 \\
\hline & & FEM & 16.1622 & 32.6827 & 52.8615 & 73.8524 & 99.9782 \\
\hline
\end{tabular}

Table 7. First natural frequencies $\Omega_{i}=\sqrt{\rho A_{1}(0) / E I_{1}(0)} L^{2} \omega_{i}$ for a two-span elastically restrained rotating Timoshenko beam, with elliptical cross section and quadratic height variation along the axis. $l_{1} / L=1 / 2 l_{2} / L=1 / 2, h_{B 1} / h_{A 1}=1 / 2, h_{B 1}^{\prime}=0$, $h_{A 2} / h_{B 1}=1 / 2, h_{B 2} / h_{A 2}=1 / 2, h_{A 2}^{\prime}=0, K_{W 1}=10, K_{\psi 1}=10, \eta=10$. 


\begin{tabular}{|c|c|c|c|c|c|c|c|}
\hline$K_{\psi d}$ & $K_{W d}$ & Method & $\Omega_{1}$ & $\Omega_{2}$ & $\Omega_{3}$ & $\Omega_{4}$ & $\Omega_{5}$ \\
\hline \multirow{10}{*}{0} & & DQM & 11.0954 & 22.8658 & 38.6771 & 56.1987 & 78.0667 \\
\hline & & FEM & 11.1149 & 22.8898 & 38.7052 & 56.2367 & 78.0931 \\
\hline & \multirow{2}{*}{0.1} & $\mathrm{DQM}$ & 14.0189 & 28.3688 & 46.9190 & 64.5736 & $8 \overline{7} . \overline{5306}$ \\
\hline & & FEM & 14.0444 & 28.3902 & 46.9506 & 64.6011 & 87.5532 \\
\hline & \multirow{2}{*}{1} & $\overline{\mathrm{DQ}} \overline{\mathrm{Q}}$ & 14.3726 & 29.3408 & 48.8766 & 68.5581 & 94.6820 \\
\hline & & FEM & 14.3992 & 29.3634 & 48.9120 & 68.5850 & 94.7094 \\
\hline & \multirow{2}{*}{10} & $\mathrm{DQM}$ & 14.4118 & 29.4478 & 49.0737 & 68.9659 & 95.3401 \\
\hline & & FEM & 14.4386 & 29.4706 & 49.1095 & 68.9930 & 95.3684 \\
\hline & \multirow{2}{*}{$\rightarrow \infty$} & $\overline{\mathrm{DQM}}$ & 14.4162 & 29.4598 & 49.0954 & 69.0107 & 95.4100 \\
\hline & & FEM & 14.4430 & 29.4826 & 49.1314 & 69.0377 & 95.4383 \\
\hline \multirow{10}{*}{1} & & DQM & 11.1299 & 23.3178 & 40.4680 & 58.6771 & 82.4016 \\
\hline & & FEM & 11.1495 & 23.3414 & 40.4960 & 58.7122 & 82.4254 \\
\hline & \multirow{2}{*}{0.1} & $\overline{\mathrm{DQM}}$ & 14.8296 & 29.6459 & 47.6815 & 64.7320 & 87.7080 \\
\hline & & FEM & 14.8556 & 29.6659 & 47.7122 & 64.7587 & 87.7309 \\
\hline & & $\mathrm{DQM}$ & 15.4691 & 31.6287 & 51.4150 & 72.0511 & 97.8483 \\
\hline & & FEM & 15.4967 & 31.6508 & 51.4509 & 72.0751 & 97.8766 \\
\hline & \multirow[b]{2}{*}{10} & $\overline{\mathrm{DQ}} \overline{\mathrm{M}}$ & 15.5418 & 31.8531 & 51.8028 & 72.9497 & $99 . \overline{1495}$ \\
\hline & & FEM & 15.5696 & 31.8754 & 51.8392 & 72.9737 & 99.1791 \\
\hline & \multirow{2}{*}{$\rightarrow \infty$} & DQM & 15.5499 & 31.8782 & 51.8452 & 73.0468 & 99.2832 \\
\hline & & FEM & 15.5778 & 31.9005 & 51.8817 & 73.0708 & 99.3129 \\
\hline \multirow{10}{*}{10} & & DQM & 11.1309 & 23.3309 & 40.5211 & 58.7582 & 82.5578 \\
\hline & & FEM & 11.1505 & 23.3545 & 40.5491 & 58.7932 & 82.5816 \\
\hline & \multirow{2}{*}{0.1} & $\overline{\mathrm{DQ}} \overline{\mathrm{M}}$ & 14.8640 & 29.7082 & 47.7217 & 64.7403 & 87.7163 \\
\hline & & FEM & 14.8900 & 29.7282 & 47.7523 & 64.7669 & 87.7393 \\
\hline & & $\mathrm{DQM}$ & 15.5170 & 31.7461 & 51.5610 & 72.2905 & 98.0837 \\
\hline & & FEM & 15.5447 & 31.7682 & 51.5970 & 72.3143 & 98.1121 \\
\hline & \multirow{2}{*}{10} & $\overline{\mathrm{DQ}} \overline{\mathrm{M}}$ & 15.5912 & 31.9764 & 51.9582 & 73.2187 & 99.4233 \\
\hline & & FEM & 15.6191 & 31.9987 & 51.9947 & 73.2426 & 99.4531 \\
\hline & \multirow{2}{*}{$\rightarrow \infty$} & DQM & 15.5996 & 32.0021 & 52.0016 & 73.3186 & 99.5601 \\
\hline & & FEM & 15.6275 & 32.0245 & 52.0381 & 73.3426 & 99.5900 \\
\hline \multirow{10}{*}{$\rightarrow \infty$} & & $\overline{\mathrm{DQM}}$ & 11.1310 & 23.3324 & 40.5272 & 58.7675 & 82.5758 \\
\hline & & FEM & 11.1506 & 23.3560 & 40.5552 & 58.8025 & 82.5996 \\
\hline & \multirow{2}{*}{0.1} & $\mathrm{DQM}$ & 14.8680 & 29.7155 & 47.7264 & 64.7412 & 87.7173 \\
\hline & & FEM & 14.8940 & 29.7355 & 47.7570 & 64.7679 & 87.7402 \\
\hline & & $\overline{\mathrm{DQ}} \overline{\mathrm{M}}$ & 15.5225 & 31.7598 & 51.5783 & 72.3191 & 98.1119 \\
\hline & & FEM & 15.5503 & 31.7819 & 51.6143 & 72.3428 & 98.1403 \\
\hline & \multirow[t]{2}{*}{10} & $\mathrm{DQM}$ & 15.5970 & 31.9908 & 51.9765 & 73.2506 & 99.4560 \\
\hline & & FEM & 15.6249 & 32.0132 & 52.0131 & 73.2745 & 99.4857 \\
\hline & \multirow{2}{*}{$\rightarrow \infty$} & $\overline{D Q M}$ & 15.6053 & 32.0166 & 52.0200 & 73.3510 & 99.5931 \\
\hline & & FEM & 15.6333 & 32.0391 & 52.0566 & 73.3749 & 99.6230 \\
\hline
\end{tabular}

Table 8. First natural frequencies $\Omega_{i}=\sqrt{\rho A_{1}(0) / E I_{1}(0)} L^{2} \omega_{i}$ for a two-span elastically restrained rotating Timoshenko beam, with elliptical cross section and quadratic height variation along the axis. $l_{1} / L=1 / 2 l_{2} / L=1 / 2, h_{B 1} / h_{A 1}=1 / 2, h_{B 1}^{\prime}=0$, $h_{A 2} / h_{B 1}=1 / 2, h_{B 2} / h_{A 2}=1 / 2, h_{A 2}^{\prime}=0, K_{W 1}=10, K_{\psi 1}=5, \eta=10$. 


\begin{tabular}{|c|c|c|c|c|c|c|c|}
\hline$K_{\psi d}$ & $K_{W d}$ & Method & $\Omega_{1}$ & $\Omega_{2}$ & $\Omega_{3}$ & $\Omega_{4}$ & $\Omega_{5}$ \\
\hline \multirow{10}{*}{0} & & DQM & 10.3650 & 21.7083 & 37.5771 & 54.9536 & 77.3897 \\
\hline & & FEM & 10.3937 & 21.7398 & 37.6121 & 54.9961 & 77.4183 \\
\hline & \multirow{2}{*}{0.1} & $\overline{\mathrm{Q}} \overline{\mathrm{M}}$ & 12.9366 & 27.1514 & 45.6367 & 63.6520 & 86.9652 \\
\hline & & FEM & 12.9736 & 27.1805 & 45.6754 & 63.6816 & 86.9900 \\
\hline & \multirow[b]{2}{*}{1} & $\overline{\mathrm{DQ}} \overline{\mathrm{M}}$ & 13.2417 & 28.0899 & $\overline{4} 7 . \overline{4960}$ & 67.6756 & $\overline{94} . \overline{0576}$ \\
\hline & & FEM & 13.2800 & 28.1204 & 47.5385 & 67.7048 & 94.0875 \\
\hline & & DQM & 13.2755 & 28.1934 & 47.6848 & 68.0876 & 94.7110 \\
\hline & & FEM & 13.3141 & 28.2242 & 47.7276 & 68.1169 & 94.7416 \\
\hline & \multirow{2}{*}{$\rightarrow \infty$} & $\overline{\mathrm{DQ}} \overline{\mathrm{M}}$ & 13.2794 & 28.2050 & $\overline{4} \overline{7} . \overline{7} 05 \overline{7}$ & 68.1329 & $9 \overline{4} . \overline{7804}$ \\
\hline & & FEM & 13.3179 & 28.2358 & 47.7485 & 68.1621 & 94.8111 \\
\hline \multirow{10}{*}{1} & & DQM & 10.3892 & 22.1044 & 39.2705 & 57.4666 & 81.7463 \\
\hline & & FEM & 10.4182 & 22.1360 & 39.3061 & 57.5054 & 81.7724 \\
\hline & \multirow[b]{2}{*}{0.1} & $\overline{\mathrm{DQM}}$ & 13.6565 & 28.4599 & 46.4416 & 63.8427 & 87.1268 \\
\hline & & FEM & 13.6946 & 28.4875 & 46.4789 & 63.8714 & 87.1519 \\
\hline & & DQM & 14.2060 & 30.3646 & 50.0205 & 71.2606 & 97.2421 \\
\hline & & FEM & 14.2462 & 30.3947 & 50.0627 & 71.2866 & 97.2727 \\
\hline & \multirow{2}{*}{10} & $\overline{\mathrm{DQ}} \overline{\mathrm{M}}$ & 14.2687 & 30.5803 & 50.3961 & 72.1634 & $\overline{98.5382}$ \\
\hline & & FEM & 14.3091 & 30.6108 & 50.4388 & 72.1896 & 98.5700 \\
\hline & \multirow{2}{*}{$\rightarrow \infty$} & $\mathrm{DQM}$ & 14.2757 & 30.6044 & 50.4372 & 72.2610 & 98.6715 \\
\hline & & FEM & 14.3161 & 30.6350 & 50.4800 & 72.2872 & 98.7035 \\
\hline \multirow{10}{*}{10} & & DQM & 10.3900 & 22.1160 & 39.3204 & 57.5486 & 81.9025 \\
\hline & & FEM & 10.4190 & 22.1475 & 39.3560 & 57.5874 & 81.9286 \\
\hline & \multirow{2}{*}{0.1} & $\mathrm{DQM}$ & 13.6869 & 28.5231 & 46.4839 & 63.8527 & 87.1344 \\
\hline & & FEM & 13.7250 & 28.5506 & 46.5212 & 63.8813 & 87.1595 \\
\hline & & $\overline{\mathrm{DQ}} \overline{\mathrm{M}}$ & 14.2479 & 30.4798 & 50.1652 & 71.5043 & $\overline{97.4786}$ \\
\hline & & FEM & 14.2881 & 30.5099 & 50.2075 & 71.5301 & 97.5092 \\
\hline & \multirow{2}{*}{10} & $\overline{\mathrm{D}} \overline{\mathrm{M}}$ & 14.3119 & 30.7011 & 50.5501 & 72.4364 & 98.8132 \\
\hline & & FEM & 14.3524 & 30.7317 & 50.5928 & 72.4624 & 98.8452 \\
\hline & \multirow{2}{*}{$\rightarrow \infty$} & DQM & 14.3191 & 30.7259 & 50.5922 & 72.5368 & 98.9498 \\
\hline & & FEM & 14.3596 & 30.7565 & 50.6350 & 72.5629 & 98.9819 \\
\hline \multirow{10}{*}{$\rightarrow \infty$} & \multirow{2}{*}{0} & DQM & 10.3901 & 22.1173 & 39.3262 & 57.5580 & 81.9205 \\
\hline & & FEM & 10.4190 & 22.1488 & 39.3618 & 57.5968 & 81.9466 \\
\hline & \multirow{2}{*}{0.1} & DQM & 13.6904 & 28.5305 & 46.4889 & 63.8539 & 87.1353 \\
\hline & & FEM & 13.7286 & 28.5580 & 46.5261 & 63.8825 & 87.1604 \\
\hline & & $\overline{\mathrm{DQ}} \overline{\mathrm{M}}$ & 14.2527 & 30.4933 & 50.1823 & 71.5333 & 97.5069 \\
\hline & & FEM & 14.2930 & 30.5235 & 50.2245 & 71.5591 & 97.5375 \\
\hline & \multirow{2}{*}{10} & DQM & 14.3169 & 30.7153 & 50.5682 & 72.4688 & 98.8460 \\
\hline & & FEM & 14.3574 & 30.7458 & 50.6110 & 72.4948 & 98.8780 \\
\hline & \multirow{2}{*}{$\rightarrow \infty$} & $\mathrm{DQM}$ & 14.3241 & 30.7401 & 50.6105 & 72.5696 & 98.9829 \\
\hline & & FEM & 14.3646 & 30.7707 & 50.6532 & 72.5957 & 99.0150 \\
\hline
\end{tabular}

Table 9. First natural frequencies $\Omega_{i}=\sqrt{\rho A_{1}(0) / E I_{1}(0)} L^{2} \omega_{i}$ for a two-span elastically restrained rotating Timoshenko beam, with elliptical cross section and quadratic height variation along the axis. $l_{1} / L=1 / 2 l_{2} / L=1 / 2, h_{B 1} / h_{A 1}=1 / 2, h_{B 1}^{\prime}=0$, $h_{A 2} / h_{B 1}=1 / 2, h_{B 2} / h_{A 2}=1 / 2, h_{A 2}^{\prime}=0, K_{W 1}=10, K_{\psi 1}=1, \eta=10$. 


\begin{tabular}{|c|c|c|c|c|c|c|c|}
\hline$K_{\psi d}$ & $K_{W d}$ & Method & $\Omega_{1}$ & $\Omega_{2}$ & $\Omega_{3}$ & $\Omega_{4}$ & $\Omega_{5}$ \\
\hline \multirow{10}{*}{0} & & DQM & 9.94295 & 21.1789 & 37.1287 & 54.4936 & 77.1422 \\
\hline & & FEM & 9.97877 & 21.2145 & 37.1668 & 54.5377 & 77.1717 \\
\hline & \multirow{2}{*}{0.1} & $\mathrm{DQM}$ & 12.3497 & 26.6244 & 45.1340 & 63.3202 & 86.7544 \\
\hline & & FEM & 12.3950 & 26.6574 & 45.1757 & 63.3506 & 86.7801 \\
\hline & \multirow{2}{*}{-1} & $\overline{\mathrm{DQ}} \overline{\mathrm{M}}$ & 12.6335 & 27.5522 & 46.9619 & 67.3571 & $\overline{93.8220}$ \\
\hline & & FEM & 12.6802 & 27.5867 & 47.0072 & 67.3872 & 93.8529 \\
\hline & \multirow{2}{*}{10} & $\mathrm{DQM}$ & 12.6651 & 27.6547 & 47.1481 & 67.7705 & 94.4732 \\
\hline & & FEM & 12.7119 & 27.6894 & 47.1937 & 67.8007 & 94.5049 \\
\hline & \multirow{2}{*}{$\rightarrow \infty$} & $\overline{\mathrm{DQM}}$ & 12.6686 & 27.6662 & $\overline{4} \overline{7} . \overline{1687}$ & 67.8159 & 94.5425 \\
\hline & & FEM & 12.7155 & 27.7009 & 47.2144 & 67.8461 & 94.5743 \\
\hline \multirow{10}{*}{1} & & DQM & 9.96265 & 21.5532 & 38.7860 & 57.0231 & 81.5044 \\
\hline & & FEM & 9.99874 & 21.5890 & 38.8248 & 57.0634 & 81.5315 \\
\hline & \multirow{2}{*}{0.1} & $\overline{\mathrm{DQM}}$ & 13.0297 & 27.9504 & 45.9575 & 63.5235 & 86.9102 \\
\hline & & FEM & 13.0763 & 27.9817 & 45.9976 & 63.5530 & 86.9362 \\
\hline & \multirow[b]{2}{*}{1} & $\mathrm{DQM}$ & 13.5409 & 29.8282 & 49.4887 & 70.9743 & 97.0122 \\
\hline & & FEM & 13.5897 & 29.8623 & 49.5335 & 71.0012 & 97.0437 \\
\hline & \multirow[b]{2}{*}{10} & $\overline{\mathrm{DQ}} \overline{\mathrm{M}}$ & 13.5994 & 30.0410 & 49.8608 & 71.8782 & $98 . \overline{3059}$ \\
\hline & & FEM & 13.6484 & 30.0754 & 49.9060 & 71.9052 & 98.3387 \\
\hline & \multirow{2}{*}{$\rightarrow \infty$} & DQM & 13.6060 & 30.0648 & 49.9016 & 71.9759 & $\overline{98.4391}$ \\
\hline & & FEM & 13.6550 & 30.0993 & 49.9469 & 72.0030 & 98.4720 \\
\hline \multirow{10}{*}{10} & & DQM & 9.96324 & 21.5641 & 38.8347 & 57.1056 & 81.6606 \\
\hline & & FEM & 9.99933 & 21.5999 & 38.8736 & 57.1459 & 81.6876 \\
\hline & \multirow{2}{*}{0.1} & $\mathrm{DQM}$ & 13.0583 & 28.0142 & 46.0008 & 63.5342 & 86.9175 \\
\hline & & FEM & 13.1049 & 28.0454 & 46.0408 & 63.5635 & 86.9436 \\
\hline & & $\mathrm{DQM}$ & 13.5802 & 29.9428 & 49.6333 & 71.2194 & 97.2490 \\
\hline & & FEM & 13.6291 & 29.9769 & 49.6781 & 71.2462 & 97.2806 \\
\hline & \multirow{2}{*}{10} & $\overline{\mathrm{D}} \overline{\mathrm{Q}} \overline{\mathrm{M}}$ & 13.6399 & 30.1611 & 50.0148 & 72.1525 & 98.5813 \\
\hline & & FEM & 13.6890 & 30.1956 & 50.0600 & 72.1794 & 98.6142 \\
\hline & \multirow{2}{*}{$\rightarrow \infty$} & DQM & 13.6467 & 30.1856 & 50.0566 & 72.2531 & 98.7177 \\
\hline & & FEM & 13.6958 & 30.2201 & 50.1018 & 72.2800 & 98.7508 \\
\hline \multirow{10}{*}{$\rightarrow \infty$} & & $\overline{\mathrm{DQM}}$ & 9.96331 & 21.5653 & 38.8403 & 57.1151 & 81.6785 \\
\hline & & FEM & 999940 & 21.6011 & 38.8792 & 57.1553 & 81.7056 \\
\hline & \multirow{2}{*}{0.1} & $\mathrm{DQM}$ & 13.0616 & 28.0216 & 46.0058 & 63.5354 & 86.9184 \\
\hline & & FEM & 13.1082 & 28.0529 & 46.0459 & 63.5648 & 86.9444 \\
\hline & \multirow[t]{2}{*}{1} & $\overline{\mathrm{DQ}} \overline{\mathrm{M}}$ & 13.5848 & 29.9563 & 49.6504 & 71.2486 & $\overline{97} . \overline{2773}$ \\
\hline & & FEM & 13.6337 & 29.9904 & 49.6952 & 71.2753 & 97.3090 \\
\hline & \multirow{2}{*}{10} & $\mathrm{DQM}$ & 13.6447 & 30.1752 & 50.0329 & 72.1851 & 98.6141 \\
\hline & & FEM & 13.6938 & 30.2097 & 50.0781 & 72.2120 & 98.6471 \\
\hline & \multirow{2}{*}{$\rightarrow \infty$} & $\overline{D Q M}$ & 13.6514 & 30.1997 & 50.0748 & 72.2860 & 98.7509 \\
\hline & & FEM & 13.7006 & 30.2342 & 50.1201 & 72.3129 & 98.7840 \\
\hline
\end{tabular}

Table 10. First natural frequencies $\Omega_{i}=\sqrt{\rho A_{1}(0) / E I_{1}(0)} L^{2} \omega_{i}$ for a two-span elastically restrained rotating Timoshenko beam, with elliptical cross section and quadratic height variation along the axis. $l_{1} / L=1 / 2 l_{2} / L=1 / 2, h_{B 1} / h_{A 1}=1 / 2, h_{B 1}^{\prime}=0$, $h_{A 2} / h_{B 1}=1 / 2, h_{B 2} / h_{A 2}=1 / 2, h_{A 2}^{\prime}=0, K_{W 1}=10, K_{\psi 1}=0.1, \eta=10$. 

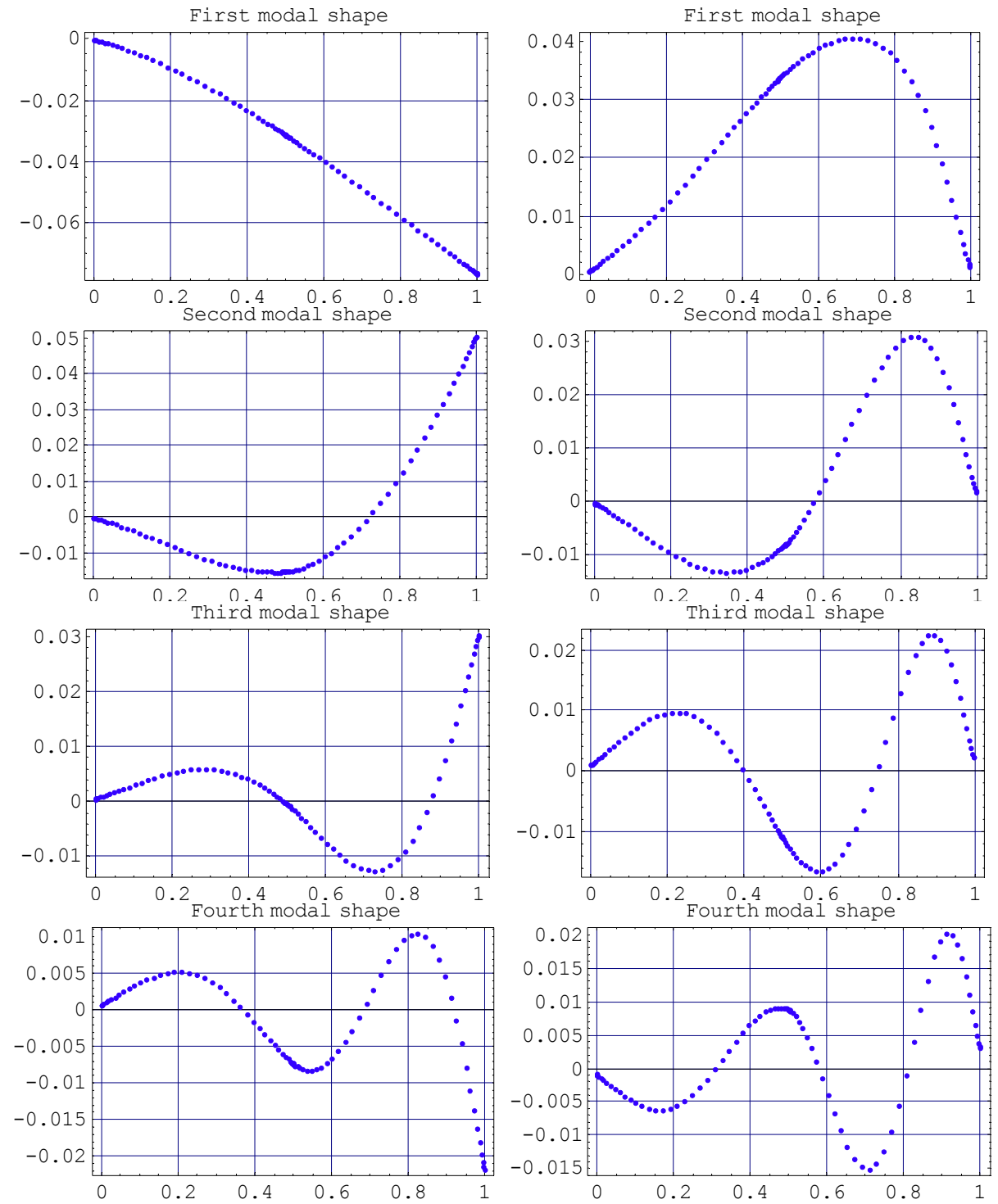

a) $K_{W 1}=10, K_{\psi 1}=5, K_{W d}=0, K_{\psi d}=0$

b) $K_{W 1}=10, K_{\psi 1}=5, K_{W d}=0.1, K_{\psi d}=1$

Fig. 4. Natural frequencies mode shapes for a two-span elastically restrained rotating Timoshenko beams, with elliptical cross section and quadratic height variation along the axis. $l_{1} / L=l_{2} / L=1 / 2 ; h_{B 1} / h_{A 1}=1 / 2 ; h_{B 1}^{\prime}=0 ; h_{A 2} / h_{B 1}=1 / 2 ; h_{B 2} / h_{A 2}=1 / 2$; $h_{A 2}^{\prime}=0 ; \eta=10$

Figure 4 shows the first four natural frequency mode shapes for beams, with two different kinds of boundary conditions: a) corresponds to $K_{W 1}=10, K_{\psi 1}=5, K_{W d}=0, K_{\psi d}=0$, while b) corresponds to $K_{W 1}=10, K_{\psi 1}=5, K_{W d}=0.1, K_{\psi d}=1$. 
The next Figures, 5 and 6, present the variation of the fundament frequency parameter $\Omega_{1}$ with the variation of the non-dimensional rotational speed $\eta$ and the spring constant $K \psi_{1}$.

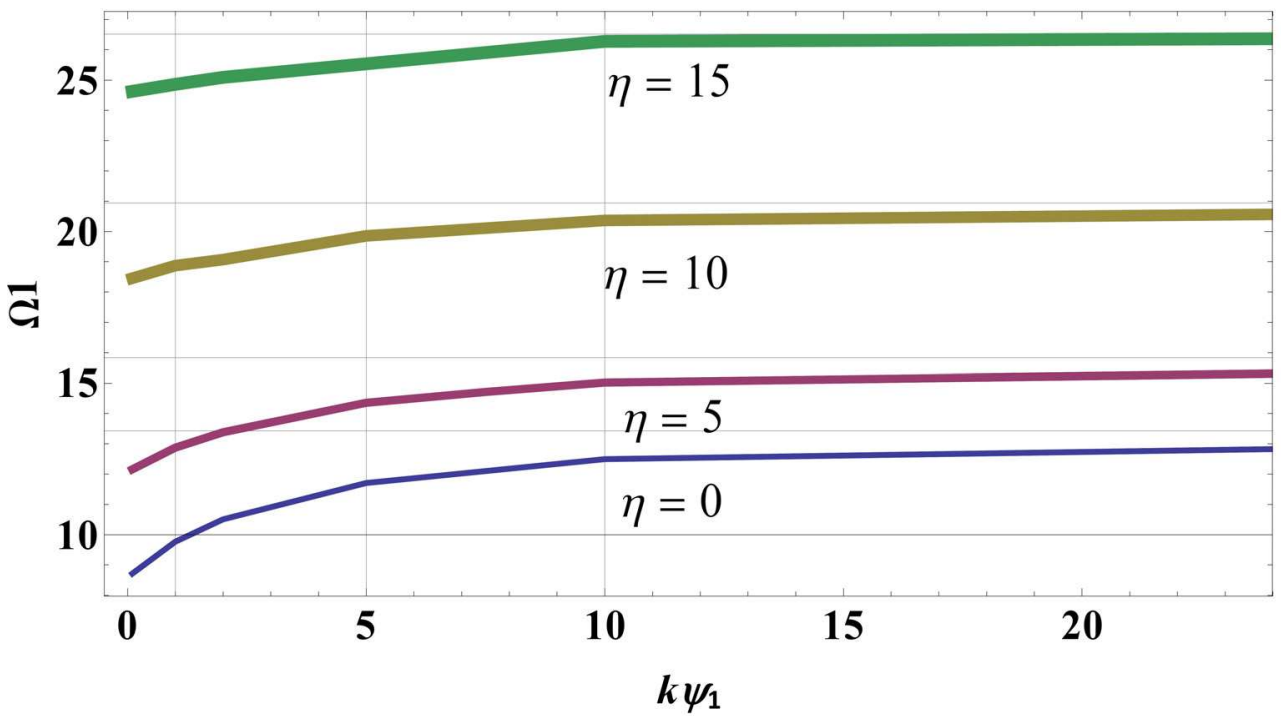

Fig. 5. The fundamental frequency coefficient $\Omega_{1}$ of a one-span elastically restrained rotating Timoshenko beam versus the spring constant parameter of the rotational spring $K_{\psi 1}$, for different rotational speed parameters $\eta . K_{w 1}=10 ; K_{w d}=1 ; K_{\psi d}=10$

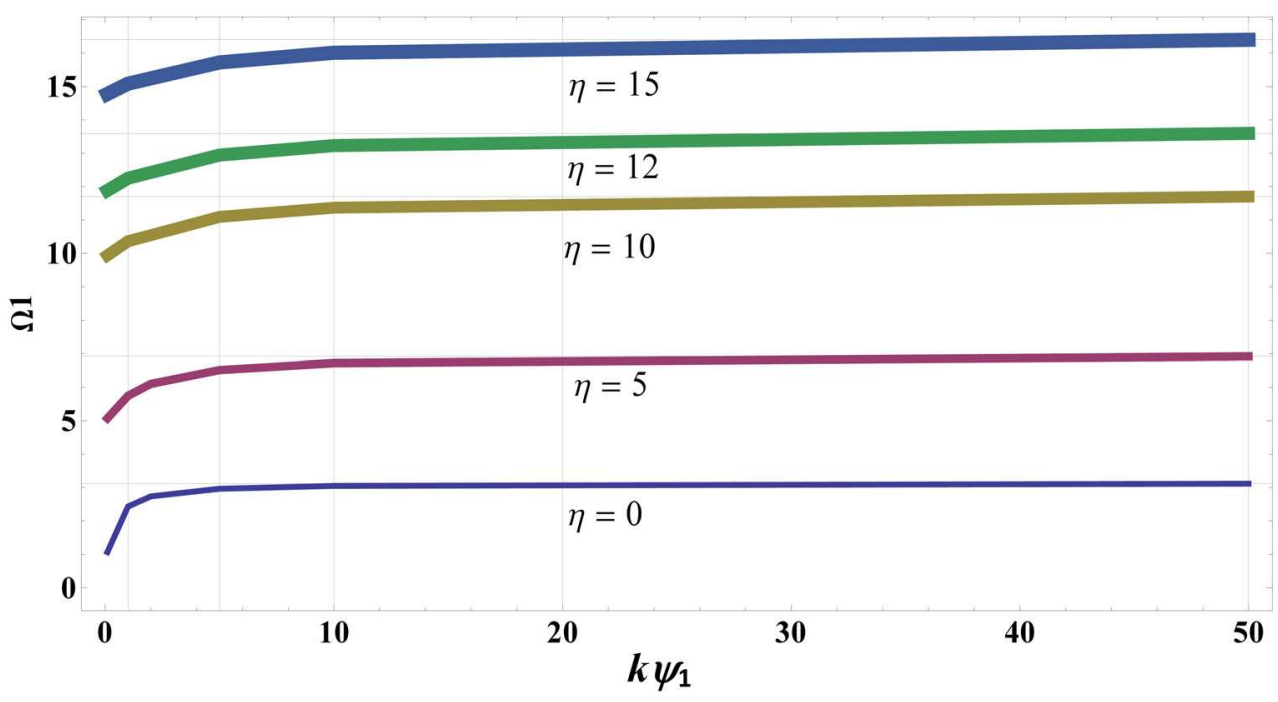

Fig. 6. The fundamental frequency coefficient $\Omega_{1}$ of a two-span elastically restrained rotating Timoshenko beam versus the spring constant parameter of the rotational spring $K_{\psi 1}$, for different rotational speed parameters $\eta$. $K_{w 1}=10 ; K_{w d}=0 ; K_{\psi d}=0$ 


\section{Conclusion}

The differential quadrature method proves to be very efficient to obtain frequencies and mode shapes of natural vibration, for the rotating Timoshenko beam model.

The versatility of the proposed beam model (variable cross section, step change in cross section, elastic restraints at both ends) allows to solve a large number of individual cases.

Something interesting to point out is that because the method directly solves two ordinary differential equations, additional restrictions are not generated. This does not happen in other methodologies, such as the dynamic stiffness method (Banerjee, 2000, 2001).

As a matter of fact, the differential quadrature method has the same advantage as the finite element method and it needs less computer memory requirements than the FEM.

In particular the present results show that the frequency coefficients vary more significantly when the translational spring stiffness changes at the end of the beam farthest from the axis of rotation $K_{\psi d}$.

\section{Appendix A}

As Shu presents in his book (Shu, 2000), the differential quadrature method, DQM, is a numerical technique for solving differential equations.

In order to obtain the DQM analog equations to the governing equations of the rotating beam and its boundary conditions, the beam domain is discretized in a grid of points using the Chebyshev - Gauss - Lobato expression, (Shu \& Chen, 1999):

$$
x_{i}=\frac{1-\cos [(i-1) \pi /(n-1)]}{2} ; i=1,2, \ldots, n
$$

where $n$ is the number of discrete points or nodes and $x_{i}$ is the coordinate of node $i$.

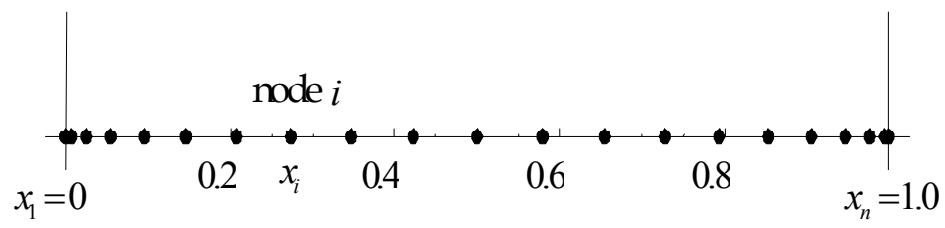

Fig. A1. Grid of $n$ points

The weighting coefficients $A_{i j}^{(1)}$ and $A_{i j}^{(2)}$, which appeared in the linear algebraic equations of quadrature (28-35), were determined using the explicit expressions cited by (Bert \& Malik, 1996).

The coefficients $A_{i j}^{(1)}$ correspond to first order derivatives and can be arranged in a square matrix of order $n$.

The matrix elements $A_{i j}^{(1)}$ with $i \neq j$, are determined by: 


$$
A_{i j}^{(1)}=\frac{\Pi\left(x_{i}\right)}{\left(x_{i}-x_{j}\right) \Pi\left(x_{j}\right)}
$$

where

$$
\Pi\left(x_{i}\right)=\prod_{v=1}^{n}\left(x_{i}-x_{v}\right) ; \Pi\left(x_{j}\right)=\prod_{v=1}^{n}\left(x_{j \neq i}-x_{v}\right) ;
$$

The coefficients $A_{i j}^{(1)}$ with $i=j$, will tend to infinity and need to be calculated in another way.

The coefficients $A_{i j}^{(2)}$ correspond to second-order derivatives and are obtained from

$$
A_{i j}^{(2)}=2\left[A_{i i}^{(1)} * A_{i j}^{(1)}-\frac{A_{i j}^{(1)}}{x_{i}-x_{j}}\right]
$$

with $i \neq j$ and $i, j=1,2,3, \ldots, n$.

Because the sum of the weighting coefficients of a row of the matrix is zero, it is easy to calculate the diagonal terms of derivatives of any order $q$, using the following expression:

$$
A_{i i}^{(q)}=-\sum_{j=1}^{n} A_{j \neq i}^{(q)}
$$

And the equations for $q$ equal to 1 and 2, corresponding to first and second order derivatives, are:

$$
A_{i i}^{(1)}=-\sum_{j=1}^{n} A_{j \neq i}^{(1)} ; \quad A_{i i}^{(2)}=-\sum_{j=1}^{n} A_{j \neq i}^{(2)}
$$

\section{Acknowledgment}

The authors gratefully acknowledge the support of the Universidad Nacional del Sur (UNS) and the Consejo Nacional de Investigaciones Científicas y Técnicas (CONICET), Argentina.

\section{References}

Bambill, D.V.; Felix, D.H. \& Rossi, R. E. (2010). Vibration analysis of rotating Timoshenko beams by means of the differential quadrature method. Structural Engineering and Mechanics, Vol. 34, No. 2, pp. 231-245, ISSN 12254568

Banerjee, J. (2000). Free vibration of centrifugally stiffened uniform and tapered beams using the dynamic stiffness method. Journal of Sound and Vibration, Vol.233, No.5, pp. 857875, ISSN 0022-460X

Banerjee, J. (2001). Dynamic stiffness formulation and free vibration analysis of centrifugally stiffened Timoshenko beam. Journal of Sound and Vibration, Vol.247, pp. 97-115, ISSN 0022-460X 
Banerjee, J.; Su, H, \& Jackson, D. (2006). Free vibration of rotating tapered beams using the dynamic stiffness method. Journal of Sound and Vibration, Vol. 298, pp. 1034-1054, ISSN 0022-460X

Bellman, R. \& Casti, J. (1971). Differential quadrature and long-term Integration. J. Math. Anal. Vol.34, pp. 235-238, ISSN 0022-247X

Bellman, R.E. \& Roth, R.S. (1986). Methods in approximation: techniques for mathematical modelling, Editorial D. Reidel Publishing Company, ISBN 9-027-72188-2, Dordrecht, Holland

Bert, C. \& Malik, M. (1996). Differential quadrature method in computational mechanics: A review. Applied Mechanics Review Vol.49, pp. 1-28, ISSN 0008-6900

Choi, S.; Wu J. \& Chou Y. (2000). Dynamic analysis of a spinning Timoshenko beam by the differential quadrature method. American Institute of Aeronautics and Astronautics Vol.38, pp. 51-856, ISSN 0001-1452

Felix, D.H.; Rossi, R. E. \& Bambill, D. V. (2008). Vibraciones transversales por el método de cuadratura diferencial de una viga Timoshenko rotante, escalonada y elásticamente vinculada, Mecánica Computacional Vol. XXVII, pp.1957-1973, ISBN 1666-6070

Felix, D. H.; Bambill, D. V. \& Rossi, R. E. (2009). Análisis de vibración libre de una viga Timoshenko escalonada, centrífugamente rigidizada, mediante el método de cuadratura diferencial, Revista Internacional de Métodos Numéricos para Cálculo y Diseño en Ingeniería. Vol. 25, No. 2, pp. 111-132, ISSN 0213-1315

Ganesh, R and Ganguli, R. (2011). Physics based basis function for vibration analysis of high speed rotating beams. Structural Engineering and Mechanics, Vol.39, No.1, pp. 21-46, ISSN 1225-4568

Gunda, J. B. \& Ganguli R. (2008). New rational interpolation functions for finite element analysis of rotating beams. International Journal of Mechanical Sciences; Vol. 50, pp. 578-588, ISSN 0020-7403

Gunda, J.B.; Singh, A.P.; Chhabra, P.S. \& Ganguli, R. (2007). Free vibration analysis of rotating tapered blades using Fourier-p superelement, Structural Engineering and Mechanics, Vol.27, No.2, pp. 243-257, ISSN 1225-4568

Kumar A. \& Ganguli R. (2009). Rotating Beams and Nonrotating Beams with Shared Eigenpair, Journal of Applied Mechanics. Vol.76. No.5, pp. 1-14, ISSN: 0021-8936

Hodges, D. H. \& Rutkowski, M. J. (1981). Free vibration analysis of rotating beams by a variable order finite method, American Institute of Aeronautics and Astronautics Journal. Vol.19, No.11, pp. 1459-1466

Lin, S. C. \& Hsiao, K. M. (2001). Vibration analysis of a rotating Timoshenko beam. Journal of Sound and Vibration. Vol. 240, pp. 303-322.

Liu, G. R. \& Wu, T. Y. (2001). Vibration analysis of beams using the generalized differential quadrature rule and domain decomposition. Journal of Sound and Vibration. Vol.246, pp.461-481, ISSN 0022-460X

Naguleswaran, S. (2004). Transverse vibration and stability of an Euler-Bernoulli beam with step change in cross-section and in axial force. Journal of Sound and Vibration. Vol.270, pp.1045-1055, ISSN 0022-460X

Özdemir, Ö. \& Kaya, M.O. (2006). Flapwise bending vibration analysis of a rotating tapered cantilever Bernoulli-Euler beam by differential transform method. Journal of Sound and Vibration. Vol.289, pp.413-420, ISSN 0022-460X 
Ozgumus, O. \& Kaya, M. O. (2010). Vibration analysis of rotating tapered Timoshenko beam using DTM. Meccanica. Vol. 45, pp. 33-42, ISSN 0025-6455

Rossi R.E. (2007). Introducción al análisis de Vibraciones con el Método de Elementos Finitos. EdiUNS, Universidad Nacional del Sur, IBSN 978-987-1171-71-2, Bahía Blanca, Argentina.

Rossi, R. E.; Gutiérrez R. H. \& Laura P. A. A. (1991). Transverse vibrations of a Timoshenko beam of nonuniform cross section elastically restrained at one end and carrying a concentrated mass at the other. J. Acoust. Soc. Am, Vol.89, pp.2456-2458.

Seon, M. H.; Benaroya, H. \& Wei, T. (1999). Dynamics of transversely vibrating beams using four engineering theories. Journal of Sound and Vibration. Vol.225, pp.35-988, ISSN 0022-460X

Singh, A.P.; Mani, V. \& Ganguli, R. (2007). Genetic programming metamodel for rotating beams, CMES - Computer modelling in Engineering and Sciences, Vol.21. No.2, pp. 133-148.

Shu, C. (2000). Differential Quadrature and Its Application in Engineering, Springer-Verlag, ISBN 1852332093, London, England

Shu, C. \& Chen, W. (1999). On optimal selection of interior points for applying discretized boundary conditions in DQ vibration analysis of beams and plates. Journal of Sound and Vibration. Vol.222, No.2, pp. 239-257, ISSN 0022-460X

Vinod, K. G., Gopalakrishnan, S. \& Ganguli, R. (2007), Free vibration and wave propagation analysis of uniform and tapered rotating beams using spectrally formulated finite elements. International Journal of Solids and Structures; Vol.44, pp. 5875-5893, ISSN 0020-7683

Yang, J. B.; Jiang, L. J. \& Chen, D. CH. (2004). Dynamic modelling and control of a rotating Euler-Bernoulli beam. Journal of Sound and Vibration. Vol.274, pp. 863-875, ISSN 0022-460X 


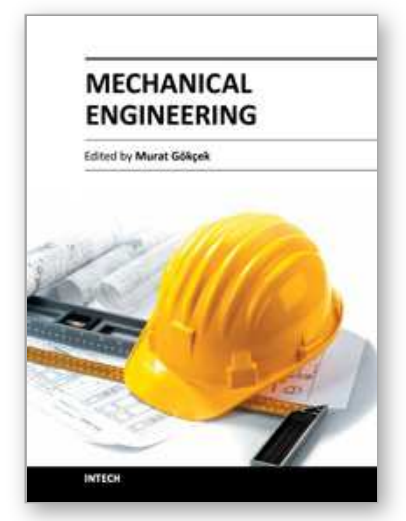

\author{
Mechanical Engineering \\ Edited by Dr. Murat Gokcek
}

ISBN 978-953-51-0505-3

Hard cover, 670 pages

Publisher InTech

Published online 11, April, 2012

Published in print edition April, 2012

The book substantially offers the latest progresses about the important topics of the "Mechanical Engineering" to readers. It includes twenty-eight excellent studies prepared using state-of-art methodologies by professional researchers from different countries. The sections in the book comprise of the following titles: power transmission system, manufacturing processes and system analysis, thermo-fluid systems, simulations and computer applications, and new approaches in mechanical engineering education and organization systems.

\title{
How to reference
}

In order to correctly reference this scholarly work, feel free to copy and paste the following:

Diana V. Bambill, Daniel H. Felix, Raúl E. Rossi and Alejandro R. Ratazzi (2012). Free Vibration Analysis of Centrifugally Stiffened Non Uniform Timoshenko Beams, Mechanical Engineering, Dr. Murat Gokcek (Ed.), ISBN: 978-953-51-0505-3, InTech, Available from: http://www.intechopen.com/books/mechanicalengineering/free-vibration-analysis-of-centrifugally-stiffened-non-uniform-timoshenko-beams

\section{INTECH}

open science | open minds

\author{
InTech Europe \\ University Campus STeP Ri \\ Slavka Krautzeka 83/A \\ 51000 Rijeka, Croatia \\ Phone: +385 (51) 770447 \\ Fax: +385 (51) 686166 \\ www.intechopen.com
}

\author{
InTech China \\ Unit 405, Office Block, Hotel Equatorial Shanghai \\ No.65, Yan An Road (West), Shanghai, 200040, China \\ 中国上海市延安西路65号上海国际贵都大饭店办公楼 405 单元 \\ Phone: +86-21-62489820 \\ Fax: $+86-21-62489821$
}


(C) 2012 The Author(s). Licensee IntechOpen. This is an open access article distributed under the terms of the Creative Commons Attribution 3.0 License, which permits unrestricted use, distribution, and reproduction in any medium, provided the original work is properly cited. 